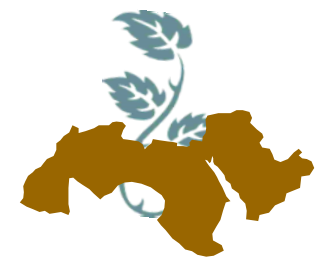

Arab Univ.

J. Agric. Sci., Ain Shams Univ., Cairo Special Issue, 26(2A), 951- 969, 2018

\title{
OPTIMAL REQUIREMENTS OF NITROGEN AND PHOSPHORUS FERTILIZATION RATES FOR STRAWBERRY NURSERIES
}

\author{
Metwally $^{1}$, A.A.; M.E. Ragab ${ }^{1}$; S.M. El-Miniawy ${ }^{1}$; S.M. Youssef ${ }^{1}$ \\ and Manal Mubarak ${ }^{2}$ \\ 1- Horticulture Dept., Fac. of Agric., Ain Shams Univ., Cairo, Egypt \\ 2- Soil Sciences Dept., Fac. of Agric., Ain Shams Univ., Cairo, Egypt
}

Keywords: Fragaria $x$ ananassa, Nursery, Fertilization, Growth, Transplant quality

\section{ABSTRACT}

Due to the expansion of strawberry nursery cultivation, transplant production has become an important industry in Egypt. Nitrogen and phosphorus are the most important nutrients affecting the number and quality of strawberry transplants. However, no empirical research exists addressing the optimal requirements of nitrogen and phosphorus fertilization rates for strawberry nurseries in Egypt, until now. This study was conducted at a private farm in Abo Ghalib, Giza Governorate, Egypt during the two successive summer seasons of 2015 and 2016 to determine the optimal requirements of nitrogen and phosphorus fertilizations for Festival strawberry cultivar nurseries via evaluating the influence of four nitrogen rates (83, 100,117 or $134 \mathrm{~kg} \mathrm{~N} /$ feddan) and five phosphorus rates $\left(38,62,74,86\right.$ or $98 \mathrm{~kg} \mathrm{P}_{2} \mathrm{O}_{5} /$ feddan) in a factorial experiment. Results revealed that increasing the rates of nitrogen and phosphorus fertilizations increased the number of main runners/mother plant and number of marketable transplants $/ \mathrm{m}^{2}$, and enhanced all vegetative growth parameters, leaf SPAD readings, leaf relative water content, crown diameter, crown carbohydrates, and leaf mineral content in both growing seasons. On the contrary, both elements had an inverse effect on leaf total soluble phenols. Thus, the study recommends to the nurserymen of Festival strawberry cultivar to apply 117 or $134 \mathrm{~kg} \mathrm{~N}$ with $98 \mathrm{~kg}$ $\mathrm{P}_{2} \mathrm{O}_{5}$ per feddan under pure sandy soil condition which gave the highest number of transplants with high quality and subsequent high quality crop in the field.

\section{INTRODUCTION}

Strawberry (Fragaria $x$ ananassa Duch.) is an important small fruit of great nutritional and medicinal value (Maas et al 1991) which belongs to the Rosaceae family. In Egypt, strawberry cultivated area was 6509 ha with an average yield of 43.55 tons ha $^{-1}$ and a total production of 283471 tons (FAOSTAT, 2014) for local consumption and exportation. This area of strawberry is cultivated with locally produced transplants. Recently, strawberry nurseries have been expanded and the licensed strawberry nurseries area was about 163 feddans in 2016 (Central Administration of Horticulture, Ministry of Agriculture and Land Reclamation, Egypt). Due to the expansion of strawberry nursery cultivation, transplant production has become an important industry in Egypt. Nitrogen and phosphorus are the most important nutrients affecting the number and quality of strawberry transplants. However, no empirical research exists addressing the optimal requirements of nitrogen and phosphorus fertilization rates for strawberry nurseries in Egypt until now.

Nitrogen is one of the important essential nutrients to the plants. It has many important functions that play roles in plant metabolic processes, development and growth of the plants. Nitrogen biologically combined with carbon, hydrogen, oxygen, and sulphur to create amino acids, which are the building units of proteins and enzymes (Epstein and Bloom, 2004). Nitrogen also is important for the chlorophyll formation which is necessary for the photosynthesis process in the plants (Uchida, 2000, Epstein and Bloom, 2004). Moreover, nitrogen encourages the uptake of other nutrients such as potassium and phosphorus (Bloom, 2015). An insufficient nitrogen supply reduces 
plant growth and leaf area and induces a decrease in the rate of photosynthesis and leaf $\mathrm{N}$ content (Deng and Woodward, 1998). On the other hand, at the excess and surplus of the nitrogen, the growth is exaggerated, with excess of leaves, causing in the susceptibility to pathogens, poor performance and low survival percentage after transplanting (Kirschbaum et al 2010).

Phosphorus also is an essential element for plant growth (Epstein and Bloom, 2004), and is an important nutrient for crop propagation, health and vigor of strawberry plants (Li et al 2010). Plants need the phosphorus for the accumulation and release of energy associated with cellular metabolism since phosphorus plays a major role in energy storage and transfer via the molecules of ADP and ATP which are the sources of the energy that drives many chemical reactions within the plants (Marschner, 2012). Also, phosphorus acts as a structural element of nucleic acids (DNA and RNA molecules) (Uchida, 2000, Marschner, 2012). The deficiency of phosphorus reduces both respiration and photosynthesis and also decreases protein and nucleic acid synthesis leading to the delaying of cell growth and subsequently decrements in plant growth (Marschner, 2012). On the other hand, excess amounts of phosphorus compete with other micronutrients like iron, manganese, and zinc resulting in leaf chlorosis and decreased plant growth (Choi and lee, 2012, Choi et al 2013)

Therefore, the current study was designed to determine the optimal requirements of nitrogen and phosphorus fertilizations for Festival strawberry cultivar nurseries via evaluating the influence of four nitrogen rates $(83,100,117$ or $134 \mathrm{~kg}$ $\mathrm{N} /$ feddan) and five phosphorus rates $(38,62,74$, 86 or $98 \mathrm{~kg} \mathrm{P}_{2} \mathrm{O}_{5} /$ feddan) in a factorial experiment.

\section{MATERIALS AND METHODS}

\section{Experimental site and cultivar}

This study was conducted at a private farm in Abo Ghalib, Giza Governorate, Egypt, during the two successive seasons of 2015 and 2016. The aim of this study was to determine the optimal requirements of nitrogen and phosphorus fertilizations for Festival strawberry cultivar nurseries, since it is an important cultivar which planted widely in Egypt for exportation as fresh or frozen fruits. The soil type was a virgin sandy soil with $\mathrm{pH}$ of 7.6 and EC of $0.8 \mathrm{mmhos} / \mathrm{cm}$. The electrical conductivity was determined in the extract of saturated soil paste according to the method mentioned by Jackson (1973). The $\mathrm{pH}$ values were measured in soil suspension (1:2.5) using $\mathrm{pH}$ meter according to the method mentioned by Black et al (1965).

\section{Fertilization treatments and cultivation}

Chicken manure at $30 \mathrm{~m}^{3} / \mathrm{feddan}$ with the initial calcium superphosphate at $250 \mathrm{~kg} / \mathrm{feddan}$ (approximately $38 \mathrm{~kg} \mathrm{P}_{2} \mathrm{O}_{5} /$ feddan) were added, three weeks before cultivation. Nursery mother transplants (Super Elite plants) were taken out from the cold storage then dipped in $0.2 \%$ Rhizolex solution for 20 minutes and planted as plugs on the $1^{\text {st }}$ and $4^{\text {th }}$ of May in the first and second season, respectively. The transplants were spaced at $1.5 \mathrm{~m}$ between plants and $1.75 \mathrm{~m}$ between rows. Flowers were continuously removed from mother plants during the first month after transplanting. Drip irrigation system in the first two months was used then the micro sprinkler irrigation system (4 $\mathrm{m} \times 5$ m) was used.

Four nitrogen fertilization rates $(83,100,117$ or $134 \mathrm{~kg} \mathrm{~N} /$ feddan) were added as ammonium nitrate fertilizer, while commercial phosphoric acid at rates of $0,40,60,80$ or $100 \mathrm{l} / \mathrm{feddan}$ to make five phosphorus fertilization rates $(38,62,74,86$ or 98 $\mathrm{kg} \mathrm{P}_{2} \mathrm{O}_{5} /$ feddan) were used. A constant rate of potassium sulfate (96 $\mathrm{kg} \mathrm{K} \mathrm{K}_{2} \mathrm{O} /$ feddan) was added. The fertilizers were added through the dripirrigation system three times a week during the nursery period.

All other agricultural practices such as runner fixation, and pest and diseases control were performed as recommended for strawberry nurseries.

\section{Experimental design}

The experiment was a factorial designed as randomized complete blocks with three replicates. Each experimental plot contained two rows each of $1.75 \mathrm{~m}$ wide and $5 \mathrm{~m}$ long (plot area $=17.5 \mathrm{~m}^{2}$ ).

\section{Data recorded}

\section{Number of runners and transplants}

Representative samples of nine mother plants with their runners and daughter plants for each experimental treatment were dug out on $1^{\text {st }}$ of October in both seasons to record the number of main runners/mother plant and number of marketable daughter transplants $/ \mathrm{m}^{2}$. 


\section{Vegetative growth characteristics}

The fresh transplants of strawberry were carefully dug out with a garden trowel to avoid the root damage. Root and plant lengths were measured. Number of leaves/plant was counted. Average leaf area was calculated as relation between area unit and fresh weight of leaves (Koller, 1972) using the following equation:

$$
\text { Leaf area }=\frac{\text { Disk area } x \text { No.of disks } x \text { fresh weight of leaves }}{\text { Fresh weight of disks }}
$$

In the laboratory, the plants were washed then root and vegetative growth fresh weights were recorded. They were dried in an oven at $70^{\circ} \mathrm{C}$ until constant weight to record the root and vegetative growth dry weights.

\section{SPAD Readings}

The leaf greenness of the obtained transplants was measured by a portable chlorophyll meter (SPAD-502, Konica Minolta Sensing, Inc., Japan). The SPAD measurements were taken at four locations on each leaf; two on each side of the midrib on the youngest fully expanded leaves of randomly selected five plants per plot and then averaged (Khan et al 2003).

\section{Total soluble phenols}

Total soluble phenol content was determined spectrophotometrically, according to the FolinCiocalteu method as described by LópezArnaldos et al (2001), using gallic acid (0-1000 $\mu \mathrm{M})$ as the standard. The absorbance at $765 \mathrm{~nm}$ of reaction media was determined using a Multiskan $^{\mathrm{TM}}$ GO Microplate Spectrophotometer after a 3 hour-incubation period at room temperature in the dark. Analysis was done in triplicate for each sample. Total soluble phenol content was expressed as gallic acid equivalents (GAE) per gram dry weight.

\section{Leaf relative water content (RWC)}

Leaf relative water content (RWC) was measured on fully expanded leaves according to the method of Barrs and Weatherley (1962). Fresh weight (FW) was immediately recorded, and then leaves were immediately soaked for 4 hours in distilled water at room temperature under a constant light and saturated humidity to record turgid weight (TW). The samples were then dried for 24 hours at $80 \stackrel{\circ}{\circ}$ for recording dry weight (DW). Relative water content (RWC) was calculated by the following formula:

$$
R W C(\%)=\frac{F W-D W}{T W-D W} \times 100
$$

\section{Leaf membrane stability index (LMSI)}

Leaf membrane stability index (LMSI) was determined by the method of Sairam et al (1997). Leaf disks $(200 \mathrm{mg})$ were taken in two sets of test tubes containing $10 \mathrm{ml}$ of distilled water. One set was kept at $40^{\circ} \mathrm{C}$ in a water bath for 30 minutes and electrical conductivity (C1) was measured. The second set was incubated at $100^{\circ} \mathrm{C}$ for 15 minutes and electrical conductivity (C2) was measured. The MSI was calculated according to the following formula:

$$
\operatorname{MSI}(\%)=\left(1-\frac{C 1}{C 2}\right) \times 100
$$

Crown diameter and crown carbohydrate determination

Average diameter of crowns was measured using caliper. Total carbohydrates of crowns were determined using phenol sulphuric acid method (DuBois et al 1956).

\section{Mineral analysis of leaves}

Leaf samples were dried at $70 \mathrm{C}^{\circ}$ in an oven until constant weight then pulverized to pass a 1 $\mathrm{mm}$ sieve. Dry samples of $0.1 \mathrm{~g}$ were taken and digested using the wet digestion method by using a mixture of sulphuric acid $98 \%$ and hydrogen peroxide $30 \%$ as described by Thomas et al (1967).

All the studied elements were assayed in the digest extract of the plant samples. Kjeldahl method was used to determine total nitrogen as described by Chapman and Pratt (1961). Spectrophotometer was used to measure phosphorus content using the ascorbic acid method (AOAC, 2005). Flame photometer was used to measure potassium as described by Page et al (1982). Calcium and magnesium were measured by the Versenate (EDTA) method as mentioned by Cheng and Bray (1951). 


\section{Statistical analysis}

The statistical analysis was conducted using the CoStat package program (Version 6.303; CoHort Software, USA). Data were subjected to analysis of variance (ANOVA). The differences among means of data were compared by Duncan's Multiple Range Test (Waller and Duncan, 1969). All statistical determinations were made at $P \leq 0.05$.

\section{RESULTS AND DISCUSSION}

\section{Number of runners and transplants}

Data in Table (1) clearly show that the applications of 117 or $134 \mathrm{~kg} \mathrm{~N} / \mathrm{feddan}$ gave the highest significant number of main runners/mother plant and number of marketable transplants $/ \mathrm{m}^{2}$ in both growing seasons. On the contrary, the application of $83 \mathrm{Kg} \mathrm{N} /$ feddan gave the lowest significant ones. These results are in a good agreement with the previous studies which reported that the high rates of nitrogen fertilization increased the number of runners and daughter plants of strawberry (Blatt and Crouse, 1970, Rodgers et al 1985, Li et al 2010). Similarly, increasing the nitrogen concentration in the nutrient solution augmented the number of runners produced in Fragaria chiloensis (Alpert, 1991, Tworkoski et al 2001). These findings may be attributed to the increments in the endogenous hormones as mentioned by Jang et al (2008) who reported that the elevated nitrogen fertilization rates increased GAs contents which enhanced the growth and development of the rice plants. The increases in the endogenous hormones especially GAs led to increases in the number of runners produced from mother plants and consequently increased the number of daughter plants in the strawberry nursery. In this connection, exogenous GA3 spraying on strawberry plants in the nurseries increased the number of transplants produced and enhanced the transplant quality (Ragab, 1996). Moreover, the increments in number of runners and number of daughter plants may be imputed to the role of nitrogen in the chlorophyll formation which improved the photosynthesis process (Uchida, 2000, Leghari et al 2016) and consequently increased the total carbohydrates which accumulated in the crown and roots of the mother plant. Also, nitrogen is essential for amino acids such as tryptophan which is an important component for biosynthesis of auxins (Zhao, 2012, Wang et al 2015) which regulate nearly all aspects of plant growth and development. In addition, nitro- gen encourages the uptake and utilization of other nutrients including potassium and phosphorous and controls overall growth of plant (Bloom, 2015).

Concerning the phosphorus fertilization, the applications of 86 or $98 \mathrm{~kg} \mathrm{P}_{2} \mathrm{O}_{5} /$ feddan gave the highest significant numbers of main runners and number of transplants, while the application of 38 $\mathrm{kg} \mathrm{P}_{2} \mathrm{O}_{5} /$ feddan gave the lowest ones in both seasons. Previous studies reported that high phosphorus rates increased the number of runners and daughter plants, while the excessive phosphorus nutrition could harm the nursery productivity since it reduced the numbers of runners and daughter plants, and increased the number of dead runners; suggesting that too much soil phosphorus may prohibit nursery plant vegetative propagation and macronutrient acquisition ( $\mathbf{L i}$ et al 2009, 2010, 2014). These increments in the numbers of runners and daughter plants may be attributed to the roles of phosphorus in storing and transfer of energy during photosynthesis process (Marschner, 2012). Also, phosphorus acts as a structural element of nucleic acids (DNA and RNA molecules) (Uchida, 2000, Marschner, 2012).

The interactive effects of the applications of nitrogen and phosphorus fertilizations were nonsignificant on the number of main runners/mother in both growing seasons. On the contrary, high rates of nitrogen with high rates of phosphorus fertilizations exhibited significant increases in number of marketable transplants $/ \mathrm{m}^{2}$. The applications of $117 \mathrm{~kg} \mathrm{~N}$ with 86 or $98 \mathrm{~kg} \mathrm{P}_{2} \mathrm{O}_{5} / \mathrm{feddan}$, and $134 \mathrm{~kg} \mathrm{~N}$ with 74,86 or $98 \mathrm{~kg} \mathrm{P}_{2} \mathrm{O}_{5} /$ feddan were the most effective applications. These increments in the number of transplants may be attributed to the synergistic functions of both elements upon the physiological and biochemical processes in the plants.

\section{Vegetative growth of plants}

Increasing the rates of nitrogen fertilization increased all vegetative growth parameters of strawberry transplants (Tables 2 and 3). The applications of 117 or $134 \mathrm{~kg} \mathrm{~N} /$ feddan gave the highest values of all recorded vegetative growth parameters, i.e. root and plant lengths, number of leaves/plant, average leaf area (Table 2) and fresh and dry weights of roots and vegetative growth (Table 3) in both growing seasons. The fertilization at $134 \mathrm{~kg} \mathrm{~N} /$ feddan gave the highest significant values of all aforementioned recorded data. On the other hand, the $83 \mathrm{~kg} \mathrm{~N} /$ feddan application gave the lowest ones in both seasons. 

for strawberry nurseries

Table 1. Effect of nitrogen and phosphorus fertilization rates on number of main runners/mother plant and number of marketable transplants $/ \mathrm{m}^{2}$ of strawberry cv. Festival in 2015 and 2016 seasons

\begin{tabular}{|c|c|c|c|c|c|}
\hline & \multirow{2}{*}{ Fertilization rates } & \multicolumn{2}{|c|}{$\begin{array}{l}\text { Number of main } \\
\text { runners/mother plant }\end{array}$} & \multicolumn{2}{|c|}{$\begin{array}{c}\text { Number of marketable } \\
\text { transplants } / \mathrm{m}^{2}\end{array}$} \\
\hline & & $1^{\text {st }}$ season & $2^{\text {nd }}$ season & $1^{\text {st }}$ season & $2^{\text {nd }}$ season \\
\hline \multicolumn{6}{|c|}{ Nitrogen fertilization } \\
\hline \multirow{4}{*}{\multicolumn{2}{|c|}{$\begin{array}{l}83 \mathrm{~kg} \mathrm{~N} / \text { feddan } \\
100 \mathrm{~kg} \mathrm{~N} / \text { feddan } \\
117 \mathrm{~kg} \mathrm{~N} / \text { feddan } \\
134 \mathrm{~kg} \mathrm{~N} / \text { feddan }\end{array}$}} & $10.67 \mathrm{c}$ & $9.13 \mathrm{c}$ & $125.87 \mathrm{c}$ & $127.73 \mathrm{c}$ \\
\hline & & $12.40 \mathrm{~b}$ & $11.47 \mathrm{~b}$ & $136.73 b$ & $150.27 b$ \\
\hline & & $14.27 \mathrm{a}$ & $12.93 \mathrm{a}$ & $150.13 a$ & $163.07 \mathrm{a}$ \\
\hline & & $14.67 \mathrm{a}$ & $13.50 \mathrm{a}$ & $152.47 \mathrm{a}$ & $165.07 \mathrm{a}$ \\
\hline \multicolumn{6}{|c|}{ Phosphorus fertilization } \\
\hline \multirow{5}{*}{\multicolumn{2}{|c|}{$\begin{array}{l}38 \mathrm{~kg} \mathrm{P}_{2} \mathrm{O}_{5} / \text { feddan } \\
62 \mathrm{~kg} \mathrm{P}_{2} \mathrm{O}_{5} / \text { feddan } \\
74 \mathrm{~kg} \mathrm{P}_{2} \mathrm{O}_{5} / \text { feddan } \\
86 \mathrm{~kg} \mathrm{P}_{2} \mathrm{O}_{5} / \text { feddan } \\
98 \mathrm{~kg} \mathrm{P}_{2} \mathrm{O}_{5} / \text { feddan }\end{array}$}} & $11.92 \mathrm{~d}$ & $10.46 \mathrm{c}$ & $131.83 \mathrm{~d}$ & $145.00 \mathrm{~d}$ \\
\hline & & $12.50 \mathrm{~cd}$ & $11.04 \mathrm{c}$ & $137.58 \mathrm{c}$ & $149.67 \mathrm{c}$ \\
\hline & & $13.00 \mathrm{bc}$ & $11.88 b$ & $142.92 b$ & $152.58 \mathrm{bc}$ \\
\hline & & $13.50 \mathrm{ab}$ & $12.54 \mathrm{ab}$ & $145.83 a b$ & $153.67 \mathrm{ab}$ \\
\hline & & $14.08 \mathrm{a}$ & $12.88 \mathrm{a}$ & $148.33 \mathrm{a}$ & $156.75 \mathrm{a}$ \\
\hline \multicolumn{6}{|c|}{ Nitrogen fertilization $X$ phosphorus fertilization interactions } \\
\hline \multirow{5}{*}{$\begin{array}{l}\frac{c}{\pi} \\
\frac{0}{0} \\
\frac{d}{2} \\
z \\
\frac{i}{z} \\
\frac{1}{m} \\
\infty\end{array}$} & $38 \mathrm{~kg} \mathrm{P}_{2} \mathrm{O}_{5} / \mathrm{feddan}$ & $9.67 \mathrm{a}$ & $7.33 \mathrm{a}$ & $113.00 \mathrm{~h}$ & $120.00 \mathrm{i}$ \\
\hline & $62 \mathrm{~kg} \mathrm{P}_{2} \mathrm{O}_{5} /$ feddan & $10.00 \mathrm{a}$ & $8.00 \mathrm{a}$ & $124.67 \mathrm{~g}$ & $124.33 \mathrm{hi}$ \\
\hline & 74 kg $\mathrm{P}_{2} \mathrm{O}_{5} /$ feddan & $10.67 \mathrm{a}$ & $9.33 \mathrm{a}$ & $127.33 \mathrm{fg}$ & $131.00 \mathrm{f}-\mathrm{h}$ \\
\hline & $86 \mathrm{~kg} \mathrm{P}_{2} \mathrm{O}_{5} / \mathrm{feddan}$ & $11.33 \mathrm{a}$ & $10.33 \mathrm{a}$ & $131.33 \mathrm{fg}$ & $129.67 \mathrm{gh}$ \\
\hline & $98 \mathrm{~kg} \mathrm{P}_{2} \mathrm{O}_{5} / \mathrm{feddan}$ & $11.67 \mathrm{a}$ & $10.67 \mathrm{a}$ & $133.00 \mathrm{f}$ & $133.67 \mathrm{fg}$ \\
\hline \multirow{5}{*}{ 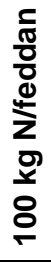 } & $38 \mathrm{~kg} \mathrm{P}_{2} \mathrm{O}_{5} /$ feddan & $11.00 \mathrm{a}$ & $10.33 \mathrm{a}$ & $125.00 \mathrm{~g}$ & 138.67 ef \\
\hline & $62 \mathrm{~kg} \mathrm{P}_{2} \mathrm{O}_{5} /$ feddan & $11.67 \mathrm{a}$ & $10.67 \mathrm{a}$ & $130.00 \mathrm{fg}$ & $149.67 \mathrm{~cd}$ \\
\hline & $74 \mathrm{~kg} \mathrm{P}_{2} \mathrm{O}_{5} /$ feddan & $12.33 \mathrm{a}$ & $11.67 \mathrm{a}$ & $140.33 \mathrm{e}$ & $145.67 \mathrm{de}$ \\
\hline & $86 \mathrm{~kg} \mathrm{P}_{2} \mathrm{O}_{5} /$ feddan & $13.33 \mathrm{a}$ & $12.00 \mathrm{a}$ & $142.00 \mathrm{de}$ & $154.33 \mathrm{bc}$ \\
\hline & $98 \mathrm{~kg} \mathrm{P}_{2} \mathrm{O}_{5} /$ feddan & $13.67 \mathrm{a}$ & $12.67 \mathrm{a}$ & 146.33 c-e & $163.00 \mathrm{a}$ \\
\hline \multirow{5}{*}{ 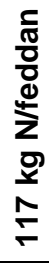 } & $38 \mathrm{~kg} \mathrm{P}_{2} \mathrm{O}_{5} / \mathrm{feddan}$ & $13.33 \mathrm{a}$ & $11.67 \mathrm{a}$ & $140.67 \mathrm{e}$ & $160.00 \mathrm{ab}$ \\
\hline & $62 \mathrm{~kg} \mathrm{P}_{2} \mathrm{O}_{5} /$ feddan & $14.00 \mathrm{a}$ & $12.33 \mathrm{a}$ & $144.00 \mathrm{de}$ & $161.00 \mathrm{ab}$ \\
\hline & $74 \mathrm{~kg} \mathrm{P}_{2} \mathrm{O}_{5} /$ feddan & $14.33 \mathrm{a}$ & $13.33 \mathrm{a}$ & $151.33 \mathrm{bc}$ & $166.00 \mathrm{a}$ \\
\hline & $86 \mathrm{~kg} \mathrm{P}_{2} \mathrm{O}_{5} / \mathrm{feddan}$ & $14.67 \mathrm{a}$ & $14.00 \mathrm{a}$ & $155.67 \mathrm{ab}$ & $164.67 \mathrm{a}$ \\
\hline & $98 \mathrm{~kg} \mathrm{P}_{2} \mathrm{O}_{5} /$ feddan & $15.00 \mathrm{a}$ & $13.33 \mathrm{a}$ & $159.00 \mathrm{a}$ & $163.67 \mathrm{a}$ \\
\hline \multirow{5}{*}{ 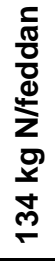 } & $38 \mathrm{~kg} \mathrm{P}_{2} \mathrm{O}_{5} /$ feddan & $13.67 \mathrm{a}$ & $12.50 \mathrm{a}$ & 148.67 b-d & $161.33 \mathrm{ab}$ \\
\hline & $62 \mathrm{~kg} \mathrm{P}_{2} \mathrm{O}_{5} /$ feddan & $14.33 \mathrm{a}$ & $13.17 \mathrm{a}$ & 151.67 bc & $163.67 \mathrm{a}$ \\
\hline & $74 \mathrm{~kg} \mathrm{P}_{2} \mathrm{O}_{5} /$ feddan & $14.67 \mathrm{a}$ & $13.17 \mathrm{a}$ & 152.67 a-c & $167.67 \mathrm{a}$ \\
\hline & $86 \mathrm{~kg} \mathrm{P}_{2} \mathrm{O}_{5} / \mathrm{feddan}$ & $14.67 \mathrm{a}$ & $13.83 \mathrm{a}$ & $154.33 \mathrm{ab}$ & $166.00 \mathrm{a}$ \\
\hline & $98 \mathrm{~kg} \mathrm{P}_{2} \mathrm{O}_{5} / \mathrm{feddan}$ & $16.00 \mathrm{a}$ & $14.83 \mathrm{a}$ & $155.00 \mathrm{ab}$ & $166.67 \mathrm{a}$ \\
\hline
\end{tabular}

Means into every group within a column for the same factor followed by the same letter are not significantly different $(P$ $\leq 0.05$ ) according to Duncan's multiple range test. 
Table 2. Effect of nitrogen and phosphorus fertilization rates on some vegetative growth characters of strawberry cv. Festival plants in 2015 and 2016 seasons

\begin{tabular}{|c|c|c|c|c|c|c|c|c|c|}
\hline \multirow{2}{*}{\multicolumn{2}{|c|}{ Fertilization rates }} & \multicolumn{2}{|c|}{$\begin{array}{l}\text { Root length } \\
\text { (cm) }\end{array}$} & \multicolumn{2}{|c|}{$\begin{array}{l}\text { Plant length } \\
(\mathrm{cm})\end{array}$} & \multicolumn{2}{|c|}{$\begin{array}{c}\text { Number of } \\
\text { leaves/plant }\end{array}$} & \multicolumn{2}{|c|}{$\begin{array}{l}\text { Average leaf area } \\
\left(\mathrm{cm}^{2}\right)\end{array}$} \\
\hline & & $\begin{array}{c}1^{\text {st }} \\
\text { season }\end{array}$ & $\begin{array}{c}2^{\text {nd }} \\
\text { season }\end{array}$ & $\begin{array}{c}1^{\text {st }} \\
\text { season }\end{array}$ & $\begin{array}{c}2^{\text {nd }} \\
\text { season }\end{array}$ & $\begin{array}{c}1^{\text {st }} \\
\text { season }\end{array}$ & $\begin{array}{c}2^{\text {nd }} \\
\text { season }\end{array}$ & $\begin{array}{c}1^{\text {st }} \\
\text { season }\end{array}$ & $\begin{array}{c}2^{\text {nd }} \\
\text { season }\end{array}$ \\
\hline \multicolumn{10}{|c|}{ Nitrogen fertilization } \\
\hline \multirow{4}{*}{\multicolumn{2}{|c|}{$\begin{array}{l}83 \mathrm{~kg} \mathrm{~N} / \text { feddan } \\
100 \mathrm{~kg} \mathrm{~N} / \mathrm{feddan} \\
117 \mathrm{~kg} \mathrm{~N} / \text { feddan } \\
134 \mathrm{~kg} \mathrm{~N} / \text { feddan }\end{array}$}} & $10.04 \mathrm{c}$ & $11.76 \mathrm{a}$ & $16.58 \mathrm{c}$ & $16.99 \mathrm{c}$ & $6.77 c$ & $6.44 d$ & $48.25 \mathrm{c}$ & $52.90 \mathrm{c}$ \\
\hline & & $10.65 b$ & $12.25 \mathrm{a}$ & $17.40 \mathrm{~b}$ & $17.91 \mathrm{~b}$ & $7.55 \mathrm{~b}$ & $7.29 \mathrm{c}$ & $51.37 \mathrm{~b}$ & $55.80 \mathrm{~b}$ \\
\hline & & $10.74 b$ & $12.09 \mathrm{a}$ & $18.28 \mathrm{a}$ & $18.69 \mathrm{a}$ & $7.81 \mathrm{ab}$ & $8.04 \mathrm{~b}$ & $53.53 \mathrm{a}$ & $57.76 \mathrm{a}$ \\
\hline & & $11.28 \mathrm{a}$ & $12.34 \mathrm{a}$ & 18.03ab & $18.67 \mathrm{a}$ & $8.16 \mathrm{a}$ & $8.65 a$ & $54.35 \mathrm{a}$ & $58.43 \mathrm{a}$ \\
\hline \multicolumn{10}{|c|}{ Phosphorus fertilization } \\
\hline \multirow{5}{*}{\multicolumn{2}{|c|}{$\begin{array}{l}38 \mathrm{~kg} \mathrm{P}_{2} \mathrm{O}_{5} / \text { feddan } \\
62 \mathrm{~kg} \mathrm{P}_{2} \mathrm{O}_{5} / \text { feddan } \\
74 \mathrm{~kg} \mathrm{P}_{2} \mathrm{O}_{5} / \text { feddan } \\
86 \mathrm{~kg} \mathrm{P}_{2} \mathrm{O}_{5} / \text { feddan } \\
98 \mathrm{~kg} \mathrm{P}_{2} \mathrm{O}_{5} / \text { feddan }\end{array}$}} & $9.43 d$ & $10.90 d$ & $16.29 b$ & $17.03 \mathrm{~b}$ & $7.13 \mathrm{c}$ & $7.18 \mathrm{c}$ & $50.11 \mathrm{c}$ & $54.25 \mathrm{c}$ \\
\hline & & $9.77 \mathrm{~d}$ & $11.17 d$ & $17.59 \mathrm{a}$ & $17.58 \mathrm{~b}$ & $7.38 \mathrm{bc}$ & $7.33 \mathrm{bc}$ & $51.41 \mathrm{~b}$ & $55.86 \mathrm{~b}$ \\
\hline & & $10.83 c$ & $11.86 \mathrm{c}$ & $17.78 \mathrm{a}$ & $18.36 \mathrm{a}$ & $7.55 \mathrm{bc}$ & $7.70 \mathrm{a}-\mathrm{c}$ & $52.03 a b$ & $56.52 \mathrm{ab}$ \\
\hline & & $11.40 \mathrm{~b}$ & $13.02 b$ & $17.95 \mathrm{a}$ & $18.44 \mathrm{a}$ & $7.80 \mathrm{ab}$ & $7.80 \mathrm{ab}$ & $52.61 \mathrm{ab}$ & $56.91 \mathrm{ab}$ \\
\hline & & $11.95 \mathrm{a}$ & $13.62 \mathrm{a}$ & $18.25 \mathrm{a}$ & $18.91 \mathrm{a}$ & $8.00 \mathrm{a}$ & $8.02 \mathrm{a}$ & $53.23 \mathrm{a}$ & $57.58 \mathrm{a}$ \\
\hline \multicolumn{10}{|c|}{ Nitrogen fertilization $\mathrm{X}$ phosphorus fertilization interactions } \\
\hline \multirow{5}{*}{ 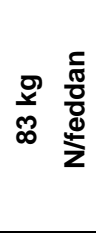 } & $38 \mathrm{~kg} \mathrm{P}_{2} \mathrm{O}_{5} /$ feddan & $8.96 \mathrm{a}$ & $11.27 \mathrm{e}-\mathrm{h}$ & $15.22 \mathrm{a}$ & $15.50 \mathrm{a}$ & $6.47 \mathrm{a}$ & $5.93 \mathrm{a}$ & $46.33 \mathrm{a}$ & $50.43 \mathrm{a}$ \\
\hline & $62 \mathrm{~kg} \mathrm{P}_{2} \mathrm{O}_{5} /$ feddan & $9.28 \mathrm{a}$ & $11.00 \mathrm{gh}$ & $16.23 \mathrm{a}$ & $16.53 \mathrm{a}$ & $6.87 \mathrm{a}$ & $6.13 \mathrm{a}$ & $47.67 \mathrm{a}$ & $52.20 \mathrm{a}$ \\
\hline & $74 \mathrm{~kg} \mathrm{P}_{2} \mathrm{O}_{5} /$ feddan & $10.45 \mathrm{a}$ & $12.07 \mathrm{~d}-\mathrm{g}$ & $16.13 \mathrm{a}$ & $17.27 \mathrm{a}$ & $6.53 \mathrm{a}$ & $6.67 \mathrm{a}$ & $48.20 \mathrm{a}$ & $53.43 \mathrm{a}$ \\
\hline & $86 \mathrm{~kg} \mathrm{P}_{2} \mathrm{O}_{5} /$ feddan & $10.67 \mathrm{a}$ & $12.20 \mathrm{~d}-\mathrm{g}$ & $17.27 \mathrm{a}$ & $17.50 \mathrm{a}$ & $6.87 \mathrm{a}$ & $6.67 \mathrm{a}$ & $49.37 \mathrm{a}$ & $53.87 \mathrm{a}$ \\
\hline & $98 \mathrm{~kg} \mathrm{P}_{2} \mathrm{O}_{5} /$ feddan & $10.83 a$ & $12.27 \mathrm{c}-\mathrm{f}$ & $18.07 \mathrm{a}$ & $18.13 \mathrm{a}$ & $7.13 \mathrm{a}$ & $6.80 \mathrm{a}$ & $49.67 \mathrm{a}$ & $54.57 \mathrm{a}$ \\
\hline \multirow{5}{*}{ 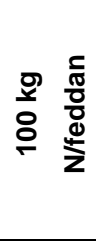 } & $38 \mathrm{~kg} \mathrm{P}_{2} \mathrm{O}_{5} /$ feddan & $9.71 \mathrm{a}$ & $11.13 \mathrm{f}-\mathrm{h}$ & $16.15 \mathrm{a}$ & $17.20 \mathrm{a}$ & $7.13 \mathrm{a}$ & $7.00 \mathrm{a}$ & $49.63 \mathrm{a}$ & $54.13 a$ \\
\hline & $62 \mathrm{~kg} \mathrm{P}_{2} \mathrm{O}_{5} /$ feddan & $10.03 \mathrm{a}$ & $12.40 \mathrm{c}-\mathrm{e}$ & $17.87 \mathrm{a}$ & $16.70 \mathrm{a}$ & $7.27 \mathrm{a}$ & $7.07 \mathrm{a}$ & 50.77 a & $54.90 \mathrm{a}$ \\
\hline & $74 \mathrm{~kg} \mathrm{P}_{2} \mathrm{O}_{5} /$ feddan & $10.83 a$ & $11.47 d-h$ & $17.37 \mathrm{a}$ & $18.30 \mathrm{a}$ & $7.53 \mathrm{a}$ & $7.33 \mathrm{a}$ & $51.57 \mathrm{a}$ & $56.10 \mathrm{a}$ \\
\hline & $86 \mathrm{~kg} \mathrm{P}_{2} \mathrm{O}_{5} /$ feddan & $10.88 \mathrm{a}$ & $12.60 \mathrm{~b}-\mathrm{d}$ & $17.37 \mathrm{a}$ & $17.90 \mathrm{a}$ & $7.87 \mathrm{a}$ & $7.47 \mathrm{a}$ & $52.33 \mathrm{a}$ & $56.60 \mathrm{a}$ \\
\hline & $98 \mathrm{~kg} \mathrm{P}_{2} \mathrm{O}_{5} /$ feddan & $11.79 \mathrm{a}$ & $13.67 \mathrm{ab}$ & $18.23 \mathrm{a}$ & $19.43 \mathrm{a}$ & $7.93 \mathrm{a}$ & $7.60 \mathrm{a}$ & $52.56 \mathrm{a}$ & $57.29 \mathrm{a}$ \\
\hline \multirow{5}{*}{ 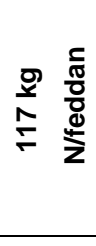 } & $38 \mathrm{~kg} \mathrm{P}_{2} \mathrm{O}_{5} /$ feddan & $9.33 \mathrm{a}$ & $10.40 \mathrm{~h}$ & $17.48 \mathrm{a}$ & $17.60 \mathrm{a}$ & $7.27 \mathrm{a}$ & $7.47 \mathrm{a}$ & $51.53 \mathrm{a}$ & $55.47 \mathrm{a}$ \\
\hline & $62 \mathrm{~kg} \mathrm{P}_{2} \mathrm{O}_{5} /$ feddan & $9.55 \mathrm{a}$ & $10.47 \mathrm{~h}$ & $18.00 \mathrm{a}$ & $18.57 \mathrm{a}$ & $7.67 \mathrm{a}$ & $7.73 \mathrm{a}$ & $53.77 \mathrm{a}$ & $57.97 \mathrm{a}$ \\
\hline & $74 \mathrm{~kg} \mathrm{P} \mathrm{P}_{5} /$ feddan & $10.67 \mathrm{a}$ & $12.33 \mathrm{c}-\mathrm{f}$ & $19.23 \mathrm{a}$ & $19.00 \mathrm{a}$ & $7.87 \mathrm{a}$ & $8.13 \mathrm{a}$ & $53.83 \mathrm{a}$ & $58.20 \mathrm{a}$ \\
\hline & $86 \mathrm{~kg} \mathrm{P}_{2} \mathrm{O}_{5} /$ feddan & $11.84 \mathrm{a}$ & $13.47 \mathrm{bc}$ & $19.03 \mathrm{a}$ & $19.13 \mathrm{a}$ & $8.07 \mathrm{a}$ & $8.40 \mathrm{a}$ & $53.87 \mathrm{a}$ & $58.27 \mathrm{a}$ \\
\hline & $98 \mathrm{~kg} \mathrm{P}_{2} \mathrm{O}_{5} /$ feddan & $12.32 \mathrm{a}$ & $13.80 \mathrm{ab}$ & $17.63 \mathrm{a}$ & $19.13 \mathrm{a}$ & $8.20 \mathrm{a}$ & $8.47 \mathrm{a}$ & $54.67 \mathrm{a}$ & $58.90 \mathrm{a}$ \\
\hline \multirow{5}{*}{ 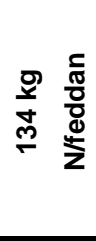 } & $38 \mathrm{~kg} \mathrm{P}_{2} \mathrm{O}_{5} /$ feddan & $9.71 \mathrm{a}$ & $10.80 \mathrm{~h}$ & $16.32 \mathrm{a}$ & $17.80 \mathrm{a}$ & $7.67 \mathrm{a}$ & $8.33 \mathrm{a}$ & $52.93 \mathrm{a}$ & $56.97 \mathrm{a}$ \\
\hline & $62 \mathrm{~kg} \mathrm{P}_{2} \mathrm{O}_{5} /$ feddan & $10.24 \mathrm{a}$ & $10.80 \mathrm{~h}$ & $18.27 \mathrm{a}$ & $18.53 \mathrm{a}$ & $7.73 \mathrm{a}$ & $8.40 \mathrm{a}$ & $53.43 \mathrm{a}$ & $58.37 \mathrm{a}$ \\
\hline & $74 \mathrm{~kg} \mathrm{P} \mathrm{P}_{5} /$ feddan & $11.39 \mathrm{a}$ & $11.57 d-h$ & $18.37 \mathrm{a}$ & $18.87 \mathrm{a}$ & $8.27 \mathrm{a}$ & $8.67 \mathrm{a}$ & $54.50 \mathrm{a}$ & $58.33 \mathrm{a}$ \\
\hline & $86 \mathrm{~kg} \mathrm{P}_{2} \mathrm{O}_{5} /$ feddan & $12.21 \mathrm{a}$ & $13.80 a b$ & $18.13 \mathrm{a}$ & $19.23 \mathrm{a}$ & $8.40 \mathrm{a}$ & $8.67 \mathrm{a}$ & $54.87 \mathrm{a}$ & $58.90 \mathrm{a}$ \\
\hline & $98 \mathrm{~kg} \mathrm{P}_{2} \mathrm{O}_{5} /$ feddan & $12.85 \mathrm{a}$ & $14.73 \mathrm{a}$ & $19.07 \mathrm{a}$ & $18.93 \mathrm{a}$ & $8.73 \mathrm{a}$ & $9.20 \mathrm{a}$ & $56.03 \mathrm{a}$ & $59.57 \mathrm{a}$ \\
\hline
\end{tabular}

Means into every group within a column for the same factor followed by the same letter are not significantly different $(P$ $\leq 0.05$ ) according to Duncan's multiple range test. 

for strawberry nurseries

Table 3. Effect of nitrogen and phosphorus fertilization rates on root and vegetative growth weights of strawberry cv. Festival plants in 2015 and 2016 seasons

\begin{tabular}{|c|c|c|c|c|c|c|c|c|c|}
\hline \multirow{2}{*}{\multicolumn{2}{|c|}{ Fertilization rates }} & \multicolumn{2}{|c|}{$\begin{array}{l}\text { Root fresh } \\
\text { weight } \\
\text { (g) }\end{array}$} & \multicolumn{2}{|c|}{$\begin{array}{l}\text { Root dry weight } \\
\text { (g) }\end{array}$} & \multicolumn{2}{|c|}{$\begin{array}{c}\text { Vegetative } \\
\text { growth fresh } \\
\text { weight }(\mathrm{g})\end{array}$} & \multicolumn{2}{|c|}{$\begin{array}{l}\text { Vegetative } \\
\text { growth dry } \\
\text { weight }(g)\end{array}$} \\
\hline & & $\begin{array}{c}1^{\text {st }} \\
\text { season }\end{array}$ & $\begin{array}{c}2^{\text {nd }} \\
\text { season }\end{array}$ & $\begin{array}{c}1^{\text {st }} \\
\text { season }\end{array}$ & $\begin{array}{c}2^{\text {nd }} \\
\text { season }\end{array}$ & $\begin{array}{c}1^{\text {st }} \\
\text { season }\end{array}$ & $\begin{array}{c}2^{\text {nd }} \\
\text { season }\end{array}$ & $\begin{array}{c}1^{\text {st }} \\
\text { season } \\
\end{array}$ & $\begin{array}{c}2^{\text {nd }} \\
\text { season } \\
\end{array}$ \\
\hline \multicolumn{10}{|c|}{ Nitrogen fertilization } \\
\hline \multicolumn{2}{|c|}{$83 \mathrm{~kg} \mathrm{~N} /$ feddan } & $0.86 \mathrm{c}$ & $0.82 \mathrm{c}$ & $0.282 b$ & $0.237 c$ & $9.47 d$ & $9.08 \mathrm{c}$ & $3.52 \mathrm{c}$ & $3.39 \mathrm{~d}$ \\
\hline \multicolumn{2}{|c|}{$100 \mathrm{~kg} \mathrm{~N} /$ feddan } & $0.99 \mathrm{~b}$ & $1.13 b$ & $0.324 a b$ & $0.308 \mathrm{~b}$ & $12.98 \mathrm{c}$ & $12.36 \mathrm{~b}$ & $4.07 \mathrm{~b}$ & $3.91 \mathrm{c}$ \\
\hline \multicolumn{2}{|c|}{$117 \mathrm{~kg} \mathrm{~N} / \mathrm{feddan}$} & $1.15 \mathrm{a}$ & $1.41 \mathrm{a}$ & $0.337 \mathrm{a}$ & $0.400 \mathrm{a}$ & $14.88 \mathrm{~b}$ & $14.76 \mathrm{a}$ & $4.26 \mathrm{~b}$ & $4.15 b$ \\
\hline \multicolumn{2}{|c|}{$134 \mathrm{~kg} \mathrm{~N} /$ feddan } & $1.19 \mathrm{a}$ & $1.47 \mathrm{a}$ & $0.363 \mathrm{a}$ & $0.399 \mathrm{a}$ & $16.23 \mathrm{a}$ & $15.10 \mathrm{a}$ & $4.69 \mathrm{a}$ & $4.50 \mathrm{a}$ \\
\hline \multicolumn{10}{|c|}{ Phosphorus fertilization } \\
\hline \multicolumn{2}{|c|}{$38 \mathrm{~kg} \mathrm{P}_{2} \mathrm{O}_{5} /$ feddan } & $0.88 c$ & $1.02 \mathrm{~d}$ & $0.279 \mathrm{c}$ & $0.270 \mathrm{~d}$ & $12.38 \mathrm{c}$ & $11.99 \mathrm{c}$ & $3.88 d$ & $3.64 \mathrm{~d}$ \\
\hline \multicolumn{2}{|c|}{$62 \mathrm{~kg} \mathrm{P}_{2} \mathrm{O}_{5} /$ feddan } & $0.93 c$ & $1.10 \mathrm{c}$ & $0.285 c$ & $0.295 \mathrm{~cd}$ & $12.71 \mathrm{c}$ & $12.22 \mathrm{c}$ & $3.96 \mathrm{~cd}$ & $3.87 \mathrm{c}$ \\
\hline \multicolumn{2}{|c|}{$74 \mathrm{~kg} \mathrm{P}_{2} \mathrm{O}_{5} / \mathrm{feddan}$} & $1.07 \mathrm{~b}$ & $1.16 \mathrm{c}$ & $0.328 \mathrm{bc}$ & $0.317 c$ & $13.39 b$ & $13.00 \mathrm{~b}$ & $4.15 b c$ & $3.99 \mathrm{bc}$ \\
\hline \multicolumn{2}{|c|}{$86 \mathrm{~kg} \mathrm{P}_{2} \mathrm{O}_{5} /$ feddan } & $1.13 a b$ & $1.27 \mathrm{~b}$ & $0.351 \mathrm{ab}$ & $0.365 b$ & $13.80 \mathrm{~b}$ & 13.36ab & $4.24 \mathrm{ab}$ & $4.13 a b$ \\
\hline \multicolumn{2}{|c|}{$98 \mathrm{~kg} \mathrm{P}_{2} \mathrm{O}_{5} / \mathrm{feddan}$} & $1.21 \mathrm{a}$ & $1.47 \mathrm{a}$ & $0.389 \mathrm{a}$ & $0.434 \mathrm{a}$ & $14.67 \mathrm{a}$ & $13.57 \mathrm{a}$ & $4.43 \mathrm{a}$ & $4.30 \mathrm{a}$ \\
\hline \multicolumn{10}{|c|}{ Nitrogen fertilization $\mathrm{X}$ phosphorus fertilization interactions } \\
\hline \multirow{5}{*}{ 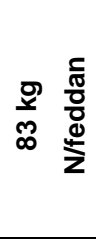 } & $38 \mathrm{~kg} \mathrm{P}_{2} \mathrm{O}_{5} /$ feddan & $0.83 \mathrm{a}$ & $0.78 \mathrm{~h}$ & $0.270 \mathrm{a}$ & $0.197 \mathrm{~h}$ & $8.13 \mathrm{a}$ & $8.19 \mathrm{a}$ & $3.23 \mathrm{a}$ & $3.04 \mathrm{a}$ \\
\hline & $62 \mathrm{~kg} \mathrm{P}_{2} \mathrm{O}_{5} /$ feddan & $0.76 \mathrm{a}$ & $0.81 \mathrm{~h}$ & $0.229 \mathrm{a}$ & $0.226 \mathrm{gh}$ & $9.02 \mathrm{a}$ & $8.07 \mathrm{a}$ & $3.35 \mathrm{a}$ & $3.21 \mathrm{a}$ \\
\hline & $74 \mathrm{~kg} \mathrm{P}_{2} \mathrm{O}_{5} /$ feddan & $0.88 \mathrm{a}$ & $0.75 \mathrm{~h}$ & $0.297 \mathrm{a}$ & $0.224 \mathrm{gh}$ & $9.53 \mathrm{a}$ & $9.40 \mathrm{a}$ & $3.47 \mathrm{a}$ & $3.36 \mathrm{a}$ \\
\hline & $86 \mathrm{~kg} \mathrm{P}_{2} \mathrm{O}_{5} / \mathrm{feddan}$ & $0.86 \mathrm{a}$ & $0.83 \mathrm{~h}$ & $0.284 \mathrm{a}$ & $0.267 \mathrm{fg}$ & $10.04 \mathrm{a}$ & $9.78 \mathrm{a}$ & $3.75 \mathrm{a}$ & $3.64 \mathrm{a}$ \\
\hline & $98 \mathrm{~kg} \mathrm{P}_{2} \mathrm{O}_{5} / \mathrm{feddan}$ & $0.95 \mathrm{a}$ & $0.91 \mathrm{gh}$ & $0.328 \mathrm{a}$ & $0.273 \mathrm{fg}$ & $10.63 \mathrm{a}$ & $9.96 \mathrm{a}$ & $3.79 \mathrm{a}$ & $3.68 \mathrm{a}$ \\
\hline \multirow{5}{*}{ 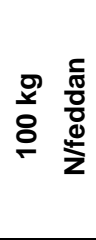 } & $38 \mathrm{~kg} \mathrm{P}_{2} \mathrm{O}_{5} /$ feddan & $0.84 \mathrm{a}$ & $1.05 \mathrm{fg}$ & $0.277 \mathrm{a}$ & $0.272 \mathrm{fg}$ & $12.15 \mathrm{a}$ & $11.38 \mathrm{a}$ & $3.73 \mathrm{a}$ & $3.51 \mathrm{a}$ \\
\hline & $62 \mathrm{~kg} \mathrm{P}_{2} \mathrm{O}_{5} /$ feddan & $0.91 \mathrm{a}$ & $1.16 \mathrm{~d}-\mathrm{f}$ & $0.313 a$ & 0.302 ef & $12.30 \mathrm{a}$ & $12.30 \mathrm{a}$ & $4.00 \mathrm{a}$ & $3.94 \mathrm{a}$ \\
\hline & $74 \mathrm{~kg} \mathrm{P}_{2} \mathrm{O}_{5} /$ feddan & $1.03 \mathrm{a}$ & 1.09 ef & $0.319 a$ & $0.285 \mathrm{fg}$ & $12.72 \mathrm{a}$ & $12.28 \mathrm{a}$ & $4.24 \mathrm{a}$ & $3.98 \mathrm{a}$ \\
\hline & $86 \mathrm{~kg} \mathrm{P}_{2} \mathrm{O}_{5} /$ feddan & $1.05 \mathrm{a}$ & 1.11 ef & $0.344 \mathrm{a}$ & $0.326 d-f$ & $13.26 \mathrm{a}$ & $13.04 \mathrm{a}$ & $4.21 \mathrm{a}$ & $4.07 \mathrm{a}$ \\
\hline & $98 \mathrm{~kg} \mathrm{P}_{2} \mathrm{O}_{5} / \mathrm{feddan}$ & $1.11 \mathrm{a}$ & $1.23 \mathrm{de}$ & $0.368 \mathrm{a}$ & $0.356 \mathrm{c}-\mathrm{e}$ & $14.48 \mathrm{a}$ & $12.81 \mathrm{a}$ & $4.17 \mathrm{a}$ & $4.04 \mathrm{a}$ \\
\hline \multirow{5}{*}{ 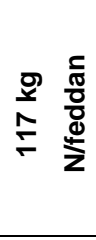 } & $38 \mathrm{~kg} \mathrm{P}_{2} \mathrm{O}_{5} /$ feddan & $0.99 \mathrm{a}$ & $1.03 \mathrm{fg}$ & $0.285 \mathrm{a}$ & 0.293 ef & $13.96 \mathrm{a}$ & $13.72 \mathrm{a}$ & $4.12 \mathrm{a}$ & $3.69 \mathrm{a}$ \\
\hline & $62 \mathrm{~kg} \mathrm{P}_{2} \mathrm{O}_{5} / \mathrm{feddan}$ & $1.02 \mathrm{a}$ & $1.17 \mathrm{~d}-\mathrm{f}$ & $0.293 \mathrm{a}$ & $0.322 d-f$ & $14.17 \mathrm{a}$ & $14.08 \mathrm{a}$ & $4.11 \mathrm{a}$ & $4.08 \mathrm{a}$ \\
\hline & $74 \mathrm{~kg} \mathrm{P}_{2} \mathrm{O}_{5} /$ feddan & $1.14 \mathrm{a}$ & $1.33 \mathrm{~cd}$ & $0.340 \mathrm{a}$ & $0.395 \mathrm{bc}$ & $15.15 \mathrm{a}$ & $15.21 \mathrm{a}$ & $4.04 \mathrm{a}$ & $4.04 \mathrm{a}$ \\
\hline & $86 \mathrm{~kg} \mathrm{P}_{2} \mathrm{O}_{5} /$ feddan & $1.23 \mathrm{a}$ & $1.56 \mathrm{~b}$ & $0.352 \mathrm{a}$ & $0.438 \mathrm{~b}$ & $15.22 \mathrm{a}$ & $15.20 \mathrm{a}$ & $4.31 \mathrm{a}$ & $4.27 \mathrm{a}$ \\
\hline & $98 \mathrm{~kg} \mathrm{P}_{2} \mathrm{O}_{5} / \mathrm{feddan}$ & $1.35 \mathrm{a}$ & $1.93 \mathrm{a}$ & $0.412 \mathrm{a}$ & $0.553 \mathrm{a}$ & $15.90 \mathrm{a}$ & $15.60 \mathrm{a}$ & $4.72 \mathrm{a}$ & $4.65 \mathrm{a}$ \\
\hline \multirow{5}{*}{ 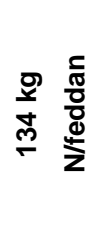 } & $38 \mathrm{~kg} \mathrm{P}_{2} \mathrm{O}_{5} / \mathrm{feddan}$ & $0.85 \mathrm{a}$ & $1.23 \mathrm{de}$ & $0.281 \mathrm{a}$ & $0.318 d-f$ & $15.30 \mathrm{a}$ & $14.66 \mathrm{a}$ & $4.43 \mathrm{a}$ & $4.31 \mathrm{a}$ \\
\hline & $62 \mathrm{~kg} \mathrm{P}_{2} \mathrm{O}_{5} /$ feddan & $1.03 \mathrm{a}$ & $1.25 \mathrm{de}$ & $0.304 \mathrm{a}$ & $0.330 \mathrm{~d}-\mathrm{f}$ & $15.36 \mathrm{a}$ & $14.44 \mathrm{a}$ & $4.38 \mathrm{a}$ & $4.23 \mathrm{a}$ \\
\hline & $74 \mathrm{~kg} \mathrm{P}_{2} \mathrm{O}_{5} /$ feddan & $1.24 \mathrm{a}$ & $1.46 \mathrm{bc}$ & $0.356 \mathrm{a}$ & $0.365 \mathrm{~cd}$ & $16.14 \mathrm{a}$ & $15.11 \mathrm{a}$ & $4.86 \mathrm{a}$ & $4.59 \mathrm{a}$ \\
\hline & $86 \mathrm{~kg} \mathrm{P}_{2} \mathrm{O}_{5} /$ feddan & $1.37 \mathrm{a}$ & $1.59 \mathrm{~b}$ & $0.426 \mathrm{a}$ & $0.428 b$ & $16.68 \mathrm{a}$ & $15.41 \mathrm{a}$ & $4.70 \mathrm{a}$ & $4.54 \mathrm{a}$ \\
\hline & $98 \mathrm{~kg} \mathrm{P}_{2} \mathrm{O}_{5} /$ feddan & $1.44 \mathrm{a}$ & $1.82 \mathrm{a}$ & $0.447 \mathrm{a}$ & $0.553 \mathrm{a}$ & $17.68 \mathrm{a}$ & $15.89 \mathrm{a}$ & $5.06 \mathrm{a}$ & $4.82 \mathrm{a}$ \\
\hline
\end{tabular}

Means into every group within a column for the same factor followed by the same letter are not significantly different $(P$ $\leq 0.05$ ) according to Duncan's multiple range test. 
These results coincide with the previous studies which reported that the number of leaves/plant (Janisch et al 2012), and plant dry mass (Kirschbaum et al 2010) of strawberry were linearly increased with increasing the nitrogen rates. Similarly, increasing the nitrogen fertilization rate led to increases in leaf area of tomato (Melton \& Dufault, 1991 and Basoccu \& Nicola, 1995), bell pepper (Dufault and Schultheis, 1994), and broccoli (Zhang et al 2017) transplants. Moreover, increases in the weight of the shoots and roots were recorded in pepper (Aloni et al 1991), tomato (Melton and Dufault, 1991, Liptay et al 1992) and lettuce (Karchi et al 1992) transplants fertilized with high rates of nitrogen. These increments in plant growth parameters of strawberry transplants may be due to the roles of nitrogen in the chlorophyll formation (Uchida, 2000, Leghari et al 2016), biosynthesis of auxins (Zhao, 2012, Wang et al 2015), the uptake and utilization of other nutrients including potassium and phosphorous (Bloom, 2015), and leaf net assimilation rate (Leghari et al 2016), which all together encourage overall plant growth and development.

As for the effect of phosphorus fertilization on strawberry transplants in the nursery, the results in Tables (2 and 3 ) revealed that phosphorus fertilization at high rates $\left(74,86\right.$ or $98 \mathrm{~kg} \mathrm{P}_{2} \mathrm{O}_{5} /$ feddan) gradually gave the highest values of growth parameters of strawberry transplants in both seasons. On the contrary, strawberry plants fertilized with $38 \mathrm{~kg} \mathrm{P}_{2} \mathrm{O}_{5} /$ feddan exhibited the lowest values. These results are in a good accordance with those obtained by Li et al (2009) who found that high phosphorus rates augmented plant height, number of leaves and leaf area of strawberry. The stimulatory effects of high phosphorus rates on the vegetative growth parameters could be attributed to the role of phosphorus in plants since phosphorus promotes root growth and development and consequently improves the uptake of nutrients including phosphorus which encourages the root elongation and all vegetative growth parameters of plants (Hill et al 2006). Moreover, the increased supply of phosphorus might have stimulated the rate of various physiological processes in the plant and led to increased growth parameters which resulted in increased number of runners and number of transplants. In this connection, data in Tables (2 and 3 ) are well correlated with data in Table (1).

Except for the root parameters (length, and fresh and dry weights of roots) in the second season, the combination between nitrogen and phos- phorus fertilizations had no significant effects on all growth parameters of strawberry transplants. However, high rates of nitrogen (117 or $134 \mathrm{~kg}$ $\mathrm{N} /$ feddan) with the highest rate of phosphorus (98 $\mathrm{kg} \mathrm{P}_{2} \mathrm{O}_{5} /$ feddan) generally gave the highest values of growth parameters. The obtained results coincide with those obtained by Nam et al (2006) who found that the dry weight of the strawberry plants cv. Nyoho cultivated under a hydroponics system increased with elevated concentrations of nitrogen and phosphorus in the fertilizer solutions. Similarly, high nitrogen rates with high rates of phosphorus increased the weights of shoots and roots and improved the transplant quality in pepper (Aloni et al 1991), tomato (Melton and Dufault, 1991), and lettuce (Karchi et al 1992). The increments in the vegetative growth characters in Tables (2 and 3 ) may be attributed to the synergistic functions of both elements upon the physiological and biochemical processes which improve overall the growth and development of the plants. In this connection, nitrogen is an essential element of all the amino acids in plant structures which are the building blocks of plant proteins (Epstein and Bloom, 2004). Furthermore, it is important in the chlorophyll formation which is necessary for the photosynthesis process in the plant (Uchida, 2000, Leghari et al 2016). In addition, phosphorus plays major roles in photosynthesis and respiration processes, since phosphorus plays a major role in energy storage and transfer (Marschner, 2012). Also, phosphorus acts as a structural element of nucleic acids (DNA and RNA molecules) (Uchida, 2000, Marschner, 2012).

\section{SPAD readings and total soluble phenols}

The applications of 117 and $134 \mathrm{~kg} \mathrm{~N} /$ feddan gave the highest significant SPAD readings, while the $83 \mathrm{~kg} \mathrm{~N} /$ feddan application gave the lowest significant ones in both growing seasons (Table 4). These results concur with those reported by García-Méndez et al (2009) who found that increasing nitrogen rates enhanced strawberry leaf chlorophyll content. The enhancement of SPAD readings may be attributed to the fact that nitrogen is a part of the enzymes associated with chlorophyll synthesis (Chapman and Barreto, 1997). On the contrary, the nitrogen fertilization showed an inverse effect on total soluble phenols. The obtained results revealed that total soluble phenols were decreased as the nitrogen fertilization increased. Similar reductions in total phenolic compounds of many plants were decreased by nitrogen fertilization (Grevesen et al 2008, Mandal et al 2008, 
Nguyen and Niemeyer, 2008, Sousa et al 2008, De Long et al 2016). These decreases in total phenolic compounds could be explained by the growth-differentiation balance (GDB) (Herms and Mattson, 1992) and carbon-nutrient balance (CNB) (Koricheva, 2002) hypotheses which suppose that when nitrogen limits plant growth, carbohydrates will accumulate in plant tissues. This increased concentration of carbohydrates will lead to an increased synthesis of carbon-based secondary metabolites such as phenolics (Massad et al 2012). Moreover, Jones and Hartley (1999) presented a protein competition model (PCM) for predicting total phenolic allocation and concentration in leaves of terrestrial higher plants in which is supposed that the accumulation of phenolics compounds is controlled by the competition between proteins and phenolics biosynthesis pathway, and thus protein and phenolic allocation are inversely correlated (Heil and Baldwin, 2002).

As for the phosphorus fertilization, the applications of 86 or $98 \mathrm{~kg} \mathrm{P}_{2} \mathrm{O}_{5} /$ feddan gave the highest significant SPAD readings in the first season, while the phosphorus fertilization did not significantly affect SPAD readings in the second season. On the contrary, as the phosphorus rates increased, total soluble phenols in strawberry leaves decreased. However, the differences were not significant in both seasons. These findings agree with the previous studies which demonstrated that phosphorus application reduced the polyphenols including the total phenolic compounds (De Long et al 2016).

In addition, the interactive effect of nitrogen and phosphorus fertilization had no significant effects neither on SPAD readings nor on total soluble phenols in strawberry leaves in both seasons.

Leaf relative water content and membrane stability index

Data in Table (5) reveal that increasing the nitrogen fertilization rates from 83 to $134 \mathrm{~kg}$ $\mathrm{N} /$ feddan increased leaf relative water content values in both seasons. However, the differences were not significant. On the contrary, increasing the nitrogen rates gradually decreased leaf membrane stability index in both seasons. In this connection, previous studies showed conflicting results on the impact of nitrogen rates on both leaf relative water content and leaf membrane stability. Lu et al (2004) found that high rates of nitrogen fertilizer increased the relative water content, while Namvar and Khandan (2015) reported that both relative water content and cell membrane stability were decreased with the high rates of nitrogen application.

Concerning the phosphorus effect, data in Table (5) show that increasing the phosphorus rates from 38 to $98 \mathrm{~kg} \mathrm{P}_{2} \mathrm{O}_{5} /$ feddan resulted in increases in relative water content and membrane stability of strawberry leaves in both seasons. However, the differences were not significant for both parameters in both growing seasons. The obtained results are consistent with the earlier studies which reported that the phosphorus fertilization levels did not affect significantly the leaf relative water content and the membrane stability index in cotton (Singh et al 2006), and maize (Naghashzadeh, 2014).

Furthermore, the interaction between nitrogen and phosphorus fertilizations did not affect both tested parameters, indicating that both factors acted independently for the two characters.

\section{Crown diameter and crown carbohydrate con- tent}

Results in Table (6) revealed that crown diameter accompanied with accumulation of carbohydrates in strawberry transplants were increased with increasing the nitrogen rates. The applications of 117 or $134 \mathrm{~kg} \mathrm{~N} /$ feddan gave the highest significant values of crown diameter and crown carbohydrate content, while the $83 \mathrm{~kg} \mathrm{~N} /$ feddan application gave the lowest significant values for both parameters in both seasons. It is well known that starch (non-soluble-nonstructural carbohydrate), glucose, fructose and sucrose (soluble nonstructural carbohydrates) are the predominant carbohydrates in the crowns of strawberry plants (MacíasRodríguez et al 2002) which are affected by the nitrogen supply. In this connection, previous studies showed conflicting results on the impact of nitrogen rates on the accumulation of carbohydrates in strawberry crowns. Carrillo-Mendoza et al (2005) found that the $\mathrm{N}$ foliar application to strawberry plants in nurseries increased the content of total reducing sugars in the crowns, while the accumulation of starch reserves and the photosynthetic rate did not affected by the treatment. However, Verpont (2003) showed that the nitrogen fertilization didn't affect the total nonstructural carbohydrates. On the contrary, high rates of nitrogen decreased the total nonstructural carbohydrates in crowns (Acuña-Maldonado and Pritts, 2008, Kirschbaum et al 2010, Kirschbaum et al 2015). 
Table 4. Effect of nitrogen and phosphorus fertilization rates on SPAD readings and total soluble phenols of strawberry cv. Festival leaves in 2015 and 2016 seasons

\begin{tabular}{|c|c|c|c|c|c|}
\hline \multirow{2}{*}{\multicolumn{2}{|c|}{ Fertilization rates }} & \multicolumn{2}{|c|}{ SPAD readings } & \multicolumn{2}{|c|}{$\begin{array}{c}\text { Total soluble phenols } \\
\text { ( } \mu \mathrm{mol} \text { eq. gallic acid /g dry } \\
\text { weight) }\end{array}$} \\
\hline & & $1^{\text {st }}$ season & $2^{\text {nd }}$ season & $1^{\text {st }}$ season & $2^{\text {nd }}$ season \\
\hline \multicolumn{6}{|c|}{ Nitrogen fertilization } \\
\hline \multicolumn{2}{|c|}{83 kg N/feddan } & $34.58 c$ & $35.34 b$ & $94.00 \mathrm{a}$ & $93.57 \mathrm{a}$ \\
\hline \multicolumn{2}{|c|}{$100 \mathrm{~kg} \mathrm{~N} /$ feddan } & $35.80 \mathrm{~b}$ & $35.84 \mathrm{~b}$ & $86.24 b$ & $86.61 \mathrm{~b}$ \\
\hline \multicolumn{2}{|r|}{$117 \mathrm{~kg} \mathrm{~N} /$ feddan } & $37.69 \mathrm{a}$ & $36.99 \mathrm{a}$ & $83.31 b$ & $81.75 b$ \\
\hline \multicolumn{2}{|c|}{$134 \mathrm{~kg} \mathrm{~N} / \mathrm{feddan}$} & $38.33 \mathrm{a}$ & $37.75 \mathrm{a}$ & $78.95 \mathrm{c}$ & $73.65 \mathrm{c}$ \\
\hline \multicolumn{6}{|c|}{ Phosphorus fertilization } \\
\hline \multirow{5}{*}{\multicolumn{2}{|c|}{$\begin{array}{l}38 \mathrm{~kg} \mathrm{P}_{2} \mathrm{O}_{5} / \text { feddan } \\
62 \mathrm{~kg} \mathrm{P} \mathrm{P}_{2} \mathrm{O}_{5} / \text { feddan } \\
74 \mathrm{~kg} \mathrm{P} \mathrm{P}_{5} / \text { feddan } \\
86 \mathrm{~kg} \mathrm{P} \mathrm{P}_{5} / \text { feddan } \\
98 \mathrm{~kg} \mathrm{P} \mathrm{O}_{5} / \text { feddan }\end{array}$}} & $35.20 \mathrm{c}$ & $35.82 \mathrm{a}$ & $88.74 \mathrm{a}$ & $86.78 \mathrm{a}$ \\
\hline & & $36.05 \mathrm{bc}$ & $35.87 \mathrm{a}$ & $86.42 \mathrm{a}$ & $86.20 \mathrm{a}$ \\
\hline & & $36.60 \mathrm{bc}$ & $36.90 \mathrm{a}$ & $85.15 \mathrm{a}$ & $83.23 \mathrm{a}$ \\
\hline & & $37.13 a b$ & $36.58 \mathrm{a}$ & $84.92 \mathrm{a}$ & $83.10 \mathrm{a}$ \\
\hline & & $38.02 \mathrm{a}$ & $37.23 \mathrm{a}$ & $82.90 \mathrm{a}$ & $80.18 \mathrm{a}$ \\
\hline \multicolumn{6}{|c|}{ Nitrogen fertilization $\mathrm{X}$ phosphorus fertilization interactions } \\
\hline \multirow{5}{*}{ 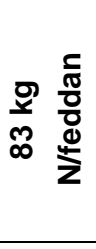 } & $38 \mathrm{~kg} \mathrm{P}_{2} \mathrm{O}_{5} /$ feddan & $32.93 \mathrm{a}$ & $34.35 \mathrm{a}$ & $97.32 \mathrm{a}$ & $96.37 \mathrm{a}$ \\
\hline & $62 \mathrm{~kg} \mathrm{P}_{2} \mathrm{O}_{5} /$ feddan & $33.90 \mathrm{a}$ & $34.05 \mathrm{a}$ & $95.66 \mathrm{a}$ & $98.50 \mathrm{a}$ \\
\hline & $74 \mathrm{~kg} \mathrm{P}_{2} \mathrm{O}_{5} /$ feddan & $35.20 \mathrm{a}$ & $36.65 \mathrm{a}$ & $91.87 \mathrm{a}$ & $93.12 \mathrm{a}$ \\
\hline & $86 \mathrm{~kg} \mathrm{P}_{2} \mathrm{O}_{5} /$ feddan & $35.03 \mathrm{a}$ & $35.52 \mathrm{a}$ & $92.33 \mathrm{a}$ & $91.75 \mathrm{a}$ \\
\hline & $98 \mathrm{~kg} \mathrm{P}_{2} \mathrm{O}_{5} / \mathrm{feddan}$ & $35.83 \mathrm{a}$ & $36.15 \mathrm{a}$ & $92.83 \mathrm{a}$ & $88.12 \mathrm{a}$ \\
\hline \multirow{5}{*}{ 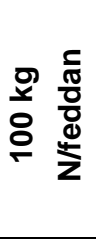 } & $38 \mathrm{~kg} \mathrm{P}_{2} \mathrm{O}_{5} / \mathrm{feddan}$ & $34.30 \mathrm{a}$ & $36.33 \mathrm{a}$ & $87.74 \mathrm{a}$ & $88.50 \mathrm{a}$ \\
\hline & $62 \mathrm{~kg} \mathrm{P}_{2} \mathrm{O}_{5} /$ feddan & $35.37 \mathrm{a}$ & $35.93 \mathrm{a}$ & $88.33 \mathrm{a}$ & $90.41 \mathrm{a}$ \\
\hline & $74 \mathrm{~kg} \mathrm{P}_{2} \mathrm{O}_{5} /$ feddan & $35.67 \mathrm{a}$ & $36.33 \mathrm{a}$ & $84.58 \mathrm{a}$ & $86.50 \mathrm{a}$ \\
\hline & $86 \mathrm{~kg} \mathrm{P}_{2} \mathrm{O}_{5} / \mathrm{feddan}$ & $36.60 \mathrm{a}$ & $35.30 \mathrm{a}$ & $88.20 \mathrm{a}$ & $87.95 \mathrm{a}$ \\
\hline & $98 \mathrm{~kg} \mathrm{P}_{2} \mathrm{O}_{5} /$ feddan & $37.07 \mathrm{a}$ & $35.30 \mathrm{a}$ & $82.37 \mathrm{a}$ & $79.70 \mathrm{a}$ \\
\hline \multirow{5}{*}{ 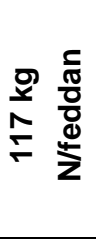 } & $38 \mathrm{~kg} \mathrm{P}_{2} \mathrm{O}_{5} /$ feddan & $36.73 \mathrm{a}$ & $36.57 \mathrm{a}$ & $86.49 \mathrm{a}$ & $84.45 \mathrm{a}$ \\
\hline & $62 \mathrm{~kg} \mathrm{P}_{2} \mathrm{O}_{5} / \mathrm{feddan}$ & $36.83 \mathrm{a}$ & $36.00 \mathrm{a}$ & $83.16 \mathrm{a}$ & $88.91 \mathrm{a}$ \\
\hline & $74 \mathrm{~kg} \mathrm{P}_{2} \mathrm{O}_{5} /$ feddan & $37.20 \mathrm{a}$ & $36.53 \mathrm{a}$ & $87.75 \mathrm{a}$ & $76.25 \mathrm{a}$ \\
\hline & $86 \mathrm{~kg} \mathrm{P}_{2} \mathrm{O}_{5} /$ feddan & $38.07 \mathrm{a}$ & $37.27 \mathrm{a}$ & $81.66 \mathrm{a}$ & $84.50 \mathrm{a}$ \\
\hline & $98 \mathrm{~kg} \mathrm{P}_{2} \mathrm{O}_{5} /$ feddan & $39.60 \mathrm{a}$ & $38.57 \mathrm{a}$ & $77.50 \mathrm{a}$ & $74.66 \mathrm{a}$ \\
\hline \multirow{5}{*}{ 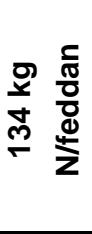 } & $38 \mathrm{~kg} \mathrm{P}_{2} \mathrm{O}_{5} /$ feddan & $36.83 \mathrm{a}$ & $36.03 \mathrm{a}$ & $83.40 \mathrm{a}$ & $77.79 \mathrm{a}$ \\
\hline & $62 \mathrm{~kg} \mathrm{P}_{2} \mathrm{O}_{5} /$ feddan & $38.10 \mathrm{a}$ & $37.50 \mathrm{a}$ & $78.54 \mathrm{a}$ & $67.00 \mathrm{a}$ \\
\hline & $74 \mathrm{~kg} \mathrm{P}_{2} \mathrm{O}_{5} /$ feddan & $38.33 \mathrm{a}$ & $38.10 \mathrm{a}$ & $76.41 \mathrm{a}$ & $77.04 \mathrm{a}$ \\
\hline & $86 \mathrm{~kg} \mathrm{P}_{2} \mathrm{O}_{5} /$ feddan & $38.80 \mathrm{a}$ & $38.23 \mathrm{a}$ & $77.50 \mathrm{a}$ & $68.20 \mathrm{a}$ \\
\hline & $98 \mathrm{~kg} \mathrm{P}_{2} \mathrm{O}_{5} /$ feddan & $39.57 \mathrm{a}$ & $38.90 \mathrm{a}$ & $78.91 \mathrm{a}$ & $78.25 \mathrm{a}$ \\
\hline
\end{tabular}

Means into every group within a column for the same factor followed by the same letter are not significantly different $(P$ $\leq 0.05$ ) according to Duncan's multiple range test. 

for strawberry nurseries

Table 5. Effect of nitrogen and phosphorus fertilization rates on relative water content and membrane stability index of strawberry cv. Festival leaves in 2015 and 2016 seasons

\begin{tabular}{|c|c|c|c|c|c|}
\hline & \multirow[t]{2}{*}{ Fertilization rates } & \multicolumn{2}{|c|}{$\begin{array}{l}\text { Leaf relative water content } \\
(\%)\end{array}$} & \multicolumn{2}{|c|}{$\begin{array}{c}\text { Membrane stability index } \\
(\%)\end{array}$} \\
\hline & & $1^{\text {st }}$ season & $2^{\text {nd }}$ season & $1^{\text {st }}$ season & $2^{\text {nd }}$ season \\
\hline \multicolumn{6}{|c|}{ Nitrogen fertilization } \\
\hline \multicolumn{2}{|r|}{83 kg N/feddan } & $63.83 \mathrm{a}$ & $64.69 \mathrm{a}$ & $74.34 \mathrm{a}$ & $74.22 \mathrm{a}$ \\
\hline \multicolumn{2}{|r|}{100 kg N/feddan } & $65.24 \mathrm{a}$ & $67.75 \mathrm{a}$ & $70.12 \mathrm{~b}$ & $70.95 b$ \\
\hline \multicolumn{2}{|r|}{117 kg N/feddan } & $65.93 \mathrm{a}$ & $68.03 \mathrm{a}$ & $66.83 \mathrm{~b}$ & $68.32 \mathrm{~b}$ \\
\hline \multicolumn{2}{|r|}{$134 \mathrm{~kg} \mathrm{~N} /$ feddan } & $68.24 \mathrm{a}$ & $69.81 \mathrm{a}$ & $63.15 \mathrm{c}$ & $65.11 \mathrm{c}$ \\
\hline \multicolumn{6}{|c|}{ Phosphorus fertilization } \\
\hline \multirow{5}{*}{\multicolumn{2}{|c|}{$\begin{array}{l}38 \mathrm{~kg} \mathrm{P}_{2} \mathrm{O}_{5} / \text { feddan } \\
62 \mathrm{~kg} \mathrm{P}_{2} \mathrm{O}_{5} / \text { feddan } \\
74 \mathrm{~kg} \mathrm{P}_{2} \mathrm{O}_{5} / \text { feddan } \\
86 \mathrm{~kg} \mathrm{P}_{2} \mathrm{O}_{5} / \text { feddan } \\
98 \mathrm{~kg} \mathrm{P}_{2} \mathrm{O}_{5} / \text { feddan }\end{array}$}} & $62.82 \mathrm{a}$ & $65.61 \mathrm{a}$ & $65.19 \mathrm{a}$ & $68.59 \mathrm{a}$ \\
\hline & & $64.05 \mathrm{a}$ & $65.91 \mathrm{a}$ & $67.97 \mathrm{a}$ & $69.02 \mathrm{a}$ \\
\hline & & $65.59 \mathrm{a}$ & $66.70 \mathrm{a}$ & $69.32 \mathrm{a}$ & $69.08 \mathrm{a}$ \\
\hline & & $67.40 \mathrm{a}$ & $68.31 \mathrm{a}$ & $69.38 \mathrm{a}$ & $69.87 \mathrm{a}$ \\
\hline & & $69.20 \mathrm{a}$ & $71.32 \mathrm{a}$ & $71.19 \mathrm{a}$ & $71.69 \mathrm{a}$ \\
\hline \multicolumn{6}{|c|}{ Nitrogen fertilization $\mathbf{X}$ phosphorus fertilization interactions } \\
\hline \multirow{5}{*}{ 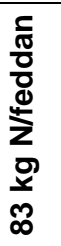 } & $38 \mathrm{~kg} \mathrm{P}_{2} \mathrm{O}_{5} /$ feddan & $64.20 \mathrm{a}$ & $60.11 \mathrm{a}$ & $71.52 \mathrm{a}$ & $73.52 \mathrm{a}$ \\
\hline & $62 \mathrm{~kg} \mathrm{P}_{2} \mathrm{O}_{5} /$ feddan & $62.68 \mathrm{a}$ & $62.21 \mathrm{a}$ & $76.34 \mathrm{a}$ & $74.22 \mathrm{a}$ \\
\hline & $74 \mathrm{~kg} \mathrm{P}_{2} \mathrm{O}_{5} /$ feddan & $62.31 \mathrm{a}$ & $65.99 \mathrm{a}$ & $75.49 \mathrm{a}$ & $74.25 \mathrm{a}$ \\
\hline & $86 \mathrm{~kg} \mathrm{P}_{2} \mathrm{O}_{5} /$ feddan & $64.67 \mathrm{a}$ & $67.21 \mathrm{a}$ & $75.00 \mathrm{a}$ & $74.28 \mathrm{a}$ \\
\hline & $98 \mathrm{~kg} \mathrm{P}_{2} \mathrm{O}_{5} / \mathrm{feddan}$ & $65.30 \mathrm{a}$ & $67.94 \mathrm{a}$ & $73.33 \mathrm{a}$ & $74.84 \mathrm{a}$ \\
\hline \multirow{5}{*}{ 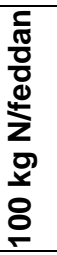 } & $38 \mathrm{~kg} \mathrm{P}_{2} \mathrm{O}_{5} /$ feddan & $60.39 \mathrm{a}$ & $68.06 \mathrm{a}$ & $68.95 \mathrm{a}$ & $72.19 \mathrm{a}$ \\
\hline & $62 \mathrm{~kg} \mathrm{P}_{2} \mathrm{O}_{5} /$ feddan & $63.45 \mathrm{a}$ & $67.84 \mathrm{a}$ & $68.85 \mathrm{a}$ & $71.26 \mathrm{a}$ \\
\hline & $74 \mathrm{~kg} \mathrm{P}_{2} \mathrm{O}_{5} /$ feddan & $65.98 \mathrm{a}$ & $67.03 \mathrm{a}$ & $71.05 \mathrm{a}$ & $70.35 \mathrm{a}$ \\
\hline & $86 \mathrm{~kg} \mathrm{P}_{2} \mathrm{O}_{5} /$ feddan & $67.74 \mathrm{a}$ & $67.86 \mathrm{a}$ & $70.40 \mathrm{a}$ & $70.48 \mathrm{a}$ \\
\hline & $98 \mathrm{~kg} \mathrm{P}_{2} \mathrm{O}_{5} /$ feddan & $68.65 \mathrm{a}$ & $67.96 \mathrm{a}$ & $71.33 \mathrm{a}$ & $70.48 \mathrm{a}$ \\
\hline \multirow{5}{*}{ 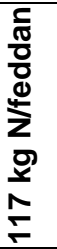 } & $38 \mathrm{~kg} \mathrm{P}_{2} \mathrm{O}_{5} /$ feddan & $64.43 \mathrm{a}$ & $66.42 \mathrm{a}$ & $59.58 \mathrm{a}$ & $64.47 \mathrm{a}$ \\
\hline & $62 \mathrm{~kg} \mathrm{P}_{2} \mathrm{O}_{5} /$ feddan & $63.60 \mathrm{a}$ & $65.16 \mathrm{a}$ & $65.26 \mathrm{a}$ & $66.91 \mathrm{a}$ \\
\hline & $74 \mathrm{~kg} \mathrm{P}_{2} \mathrm{O}_{5} /$ feddan & $65.04 \mathrm{a}$ & $65.02 \mathrm{a}$ & $69.58 \mathrm{a}$ & $68.98 \mathrm{a}$ \\
\hline & $86 \mathrm{~kg} \mathrm{P}_{2} \mathrm{O}_{5} /$ feddan & $66.80 \mathrm{a}$ & $69.27 \mathrm{a}$ & $69.94 \mathrm{a}$ & $69.77 \mathrm{a}$ \\
\hline & $98 \mathrm{~kg} \mathrm{P}_{2} \mathrm{O}_{5} /$ feddan & $69.76 \mathrm{a}$ & $74.30 \mathrm{a}$ & $69.79 \mathrm{a}$ & $71.47 \mathrm{a}$ \\
\hline \multirow{5}{*}{ 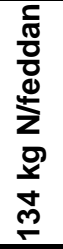 } & $38 \mathrm{~kg} \mathrm{P}_{2} \mathrm{O}_{5} /$ feddan & $62.24 \mathrm{a}$ & $67.85 \mathrm{a}$ & $60.72 \mathrm{a}$ & $64.19 \mathrm{a}$ \\
\hline & $62 \mathrm{~kg} \mathrm{P}_{2} \mathrm{O}_{5} /$ feddan & $66.46 \mathrm{a}$ & $68.44 \mathrm{a}$ & $61.41 \mathrm{a}$ & $63.69 \mathrm{a}$ \\
\hline & $74 \mathrm{~kg} \mathrm{P}_{2} \mathrm{O}_{5} /$ feddan & $69.04 \mathrm{a}$ & $68.75 \mathrm{a}$ & $61.15 \mathrm{a}$ & $62.72 \mathrm{a}$ \\
\hline & $86 \mathrm{~kg} \mathrm{P}_{2} \mathrm{O}_{5} /$ feddan & $70.40 \mathrm{a}$ & $68.91 \mathrm{a}$ & $62.17 \mathrm{a}$ & $64.96 \mathrm{a}$ \\
\hline & $98 \mathrm{~kg} \mathrm{P}_{2} \mathrm{O}_{5} /$ feddan & $73.08 \mathrm{a}$ & $75.09 \mathrm{a}$ & $70.31 \mathrm{a}$ & $69.98 \mathrm{a}$ \\
\hline
\end{tabular}

Means into every group within a column for the same factor followed by the same letter are not significantly different $(P$ $\leq 0.05$ ) according to Duncan's multiple range test. 
Table 6. Effect of nitrogen and phosphorus fertilization rates on crown diameter and crown carbohydrates of strawberry cv. Festival in 2015 and 2016 seasons

\begin{tabular}{|c|c|c|c|c|c|}
\hline \multirow{2}{*}{\multicolumn{2}{|c|}{ Fertilization rates }} & \multicolumn{2}{|c|}{$\begin{array}{c}\text { Crown diameter } \\
(\mathrm{cm})\end{array}$} & \multicolumn{2}{|c|}{$\begin{array}{l}\text { Crown carbohydrates } \\
\text { (mg/ g dry weight) }\end{array}$} \\
\hline & & $1^{\text {st }}$ season & $2^{\text {nd }}$ season & $1^{\text {st }}$ season & $2^{\text {nd }}$ season \\
\hline \multicolumn{6}{|c|}{ Nitrogen fertilization } \\
\hline \multicolumn{2}{|c|}{$83 \mathrm{~kg} \mathrm{~N} / \mathrm{feddan}$} & $0.92 \mathrm{c}$ & $0.96 \mathrm{c}$ & $174.18 \mathrm{c}$ & $176.13 \mathrm{c}$ \\
\hline \multicolumn{2}{|c|}{$100 \mathrm{~kg} \mathrm{~N} /$ feddan } & $0.99 \mathrm{~b}$ & $1.04 \mathrm{~b}$ & $194.89 \mathrm{~b}$ & $185.78 \mathrm{~b}$ \\
\hline \multicolumn{2}{|c|}{117 kg N/feddan } & $1.01 \mathrm{ab}$ & $1.11 \mathrm{a}$ & $206.62 \mathrm{a}$ & $192.53 \mathrm{a}$ \\
\hline \multicolumn{2}{|c|}{134 kg N/feddan } & $1.04 \mathrm{a}$ & $1.10 \mathrm{a}$ & $210.21 \mathrm{a}$ & $194.76 \mathrm{a}$ \\
\hline \multicolumn{6}{|c|}{ Phosphorus fertilization } \\
\hline \multirow{5}{*}{\multicolumn{2}{|c|}{$\begin{array}{l}38 \mathrm{~kg} \mathrm{P}_{2} \mathrm{O}_{5} / \text { feddan } \\
62 \mathrm{~kg} \mathrm{P} \mathrm{P}_{2} \mathrm{O}_{5} / \text { feddan } \\
74 \mathrm{~kg} \mathrm{P}_{2} \mathrm{O}_{5} / \text { feddan } \\
86 \mathrm{~kg} \mathrm{P}_{2} \mathrm{O}_{5} / \text { feddan } \\
98 \mathrm{~kg} \mathrm{P}_{2} \mathrm{O}_{5} / \text { feddan }\end{array}$}} & $0.90 \mathrm{c}$ & $0.95 d$ & 191.12 c & 181.22 c \\
\hline & & $0.94 \mathrm{c}$ & $1.02 \mathrm{c}$ & $195.78 \mathrm{~b}$ & $185.95 \mathrm{~b}$ \\
\hline & & $0.94 \mathrm{c}$ & $1.04 \mathrm{c}$ & $196.28 \mathrm{ab}$ & $188.22 a b$ \\
\hline & & $1.01 \mathrm{~b}$ & $1.10 \mathrm{~b}$ & $198.83 a b$ & $189.61 \mathrm{a}$ \\
\hline & & $1.16 \mathrm{a}$ & $1.16 \mathrm{a}$ & $200.38 \mathrm{a}$ & $191.50 \mathrm{a}$ \\
\hline \multicolumn{6}{|c|}{ Nitrogen fertilization X phosphorus fertilization interactions } \\
\hline \multirow{5}{*}{ 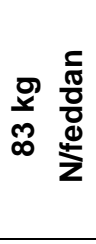 } & $38 \mathrm{~kg} \mathrm{P}_{2} \mathrm{O}_{5} /$ feddan & $0.85 \mathrm{a}$ & $0.89 \mathrm{~h}$ & $165.81 \mathrm{a}$ & $168.11 \mathrm{a}$ \\
\hline & 62 kg $\mathrm{P}_{2} \mathrm{O}_{5} /$ feddan & $0.89 \mathrm{a}$ & $0.95 \mathrm{gh}$ & $167.89 \mathrm{a}$ & $173.00 \mathrm{a}$ \\
\hline & 74 kg $\mathrm{P}_{2} \mathrm{O}_{5} /$ feddan & $0.90 \mathrm{a}$ & $0.98 \mathrm{fg}$ & $175.21 \mathrm{a}$ & $178.11 \mathrm{a}$ \\
\hline & $86 \mathrm{~kg} \mathrm{P}_{2} \mathrm{O}_{5} / \mathrm{feddan}$ & $0.92 \mathrm{a}$ & $0.99 \mathrm{e}-\mathrm{g}$ & $180.44 \mathrm{a}$ & $179.56 \mathrm{a}$ \\
\hline & $98 \mathrm{~kg} \mathrm{P}_{2} \mathrm{O}_{5} /$ feddan & $1.05 \mathrm{a}$ & $1.00 \mathrm{e}-\mathrm{g}$ & $181.56 \mathrm{a}$ & $181.89 \mathrm{a}$ \\
\hline \multirow{5}{*}{ 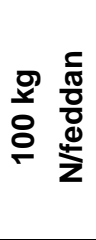 } & $38 \mathrm{~kg} \mathrm{P}_{2} \mathrm{O}_{5} /$ feddan & $0.91 \mathrm{a}$ & $0.96 \mathrm{gh}$ & $187.22 \mathrm{a}$ & $182.00 \mathrm{a}$ \\
\hline & $62 \mathrm{~kg} \mathrm{P}_{2} \mathrm{O}_{5} /$ feddan & $0.93 \mathrm{a}$ & $1.03 \mathrm{~d}-\mathrm{g}$ & $199.78 \mathrm{a}$ & $183.03 \mathrm{a}$ \\
\hline & $74 \mathrm{~kg} \mathrm{P}_{2} \mathrm{O}_{5} /$ feddan & $0.92 \mathrm{a}$ & 0.99 e-g & $193.67 \mathrm{a}$ & $186.33 \mathrm{a}$ \\
\hline & $86 \mathrm{~kg} \mathrm{P}_{2} \mathrm{O}_{5} /$ feddan & $0.99 a$ & $1.08 \mathrm{~b}-\mathrm{e}$ & $195.56 \mathrm{a}$ & $188.33 \mathrm{a}$ \\
\hline & $98 \mathrm{~kg} \mathrm{P}_{2} \mathrm{O}_{5} /$ feddan & $1.18 \mathrm{a}$ & $1.13 \mathrm{bc}$ & $198.22 \mathrm{a}$ & $189.22 \mathrm{a}$ \\
\hline \multirow{5}{*}{ 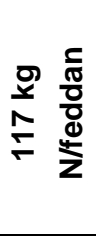 } & $38 \mathrm{~kg} \mathrm{P}_{2} \mathrm{O}_{5} /$ feddan & $0.90 \mathrm{a}$ & 0.99 e-g & $204.89 \mathrm{a}$ & 184.89 a \\
\hline & $62 \mathrm{~kg} \mathrm{P}_{2} \mathrm{O}_{5} /$ feddan & $0.94 \mathrm{a}$ & $1.07 c-f$ & $206.89 \mathrm{a}$ & $193.22 \mathrm{a}$ \\
\hline & 74 kg $\mathrm{P}_{2} \mathrm{O}_{5} /$ feddan & $0.98 \mathrm{a}$ & $1.08 \mathrm{~b}-\mathrm{e}$ & $206.78 \mathrm{a}$ & $194.00 \mathrm{a}$ \\
\hline & $86 \mathrm{~kg} \mathrm{P}_{2} \mathrm{O}_{5} /$ feddan & $1.02 \mathrm{a}$ & $1.17 \mathrm{~b}$ & $206.44 \mathrm{a}$ & $194.22 \mathrm{a}$ \\
\hline & $98 \mathrm{~kg} \mathrm{P}_{2} \mathrm{O}_{5} /$ feddan & $1.19 \mathrm{a}$ & $1.26 \mathrm{a}$ & $208.11 \mathrm{a}$ & $196.33 \mathrm{a}$ \\
\hline \multirow{5}{*}{ 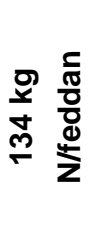 } & $38 \mathrm{~kg} \mathrm{P} \mathrm{O}_{5} /$ feddan & $0.93 \mathrm{a}$ & $0.95 \mathrm{gh}$ & $206.56 \mathrm{a}$ & $189.89 \mathrm{a}$ \\
\hline & $62 \mathrm{~kg} \mathrm{P}_{2} \mathrm{O}_{5} /$ feddan & $0.99 \mathrm{a}$ & $1.04 \mathrm{~d}-\mathrm{g}$ & $208.56 \mathrm{a}$ & $194.56 \mathrm{a}$ \\
\hline & 74 kg $\mathrm{P}_{2} \mathrm{O}_{5} /$ feddan & $0.97 \mathrm{a}$ & $1.10 \mathrm{~b}-\mathrm{d}$ & $209.44 \mathrm{a}$ & $194.44 \mathrm{a}$ \\
\hline & $86 \mathrm{~kg} \mathrm{P}_{2} \mathrm{O}_{5} /$ feddan & $1.11 \mathrm{a}$ & $1.17 \mathrm{~b}$ & $212.89 \mathrm{a}$ & $196.33 \mathrm{a}$ \\
\hline & $98 \mathrm{~kg} \mathrm{P}_{2} \mathrm{O}_{5} /$ feddan & $1.21 \mathrm{a}$ & $1.26 \mathrm{a}$ & $213.61 \mathrm{a}$ & $198.56 \mathrm{a}$ \\
\hline
\end{tabular}

Means into every group within a column for the same factor followed by the same letter are not significantly different $(P$ $\leq 0.05$ ) according to Duncan's multiple range test. 
These conflicting results may be attributed to the fact that the accumulation of carbohydrates is highly specific for individual carbohydrates and different plant tissues, and further depend on other environmental factors (Druege et al 2004).

As for the effect of phosphorus fertilization, the highest rate of phosphorus (98 $\mathrm{kg} \mathrm{P}_{2} \mathrm{O}_{5} /$ feddan) gave the highest values of crown diameters in both growing seasons, while the high rates of phosphorus applications $\left(74,86\right.$ or $98 \mathrm{~kg} \mathrm{P}_{2} \mathrm{O}_{5} /$ feddan) gave the highest values of crown carbohydrates without significant differences among them. On the other hand, the lowest rate of phosphorus application (38 $\mathrm{kg} \mathrm{P}_{2} \mathrm{O}_{5} /$ feddan) gave the lowest values of both characteristics in both seasons. These findings are in agreements with those obtained by Hermans et al (2006) who stated that under phosphorus deficiency, starch and soluble carbohydrates are expected to accumulate in the source organ (leaves) and diminish in the sink organs (in our case, crowns).

Although the interactive effects of nitrogen and phosphorus fertilizations were not significant on crown diameter in the first season and crown carbohydrates in both seasons, the applications of 117 or $134 \mathrm{~kg} \mathrm{~N} /$ feddan with $98 \mathrm{~kg} \mathrm{P}_{2} \mathrm{O}_{5} /$ feddan gave the highest values in both seasons. The increments in strawberry crowns resulted from fertilization with nitrogen and phosphorus are well related with the increments in the growth parameters Tables (2 and 3), since carbohydrate is important in energy production which is necessary for cell activities.

\section{Leaf mineral analysis}

Data in Table (7) show that the 117 and $134 \mathrm{~kg}$ $\mathrm{N} /$ feddan applications gave the highest percentages of nitrogen, phosphorus, potassium and magnesium in leaves, and the lowest calcium percentage in both seasons. However, the $83 \mathrm{~kg}$ $\mathrm{N} /$ feddan gave the highest calcium percentage in leaves; but it gave the lowest percentages of nitrogen, phosphorus, potassium and magnesium in both seasons. These results agree with those reported by Verpont (2003), García-Méndez et al (2009), Kirschbaum et al (2010), and Kirschbaum et al (2015) that the increase of nitrogen rates increased nitrogen concentration in leaves, roots and crowns. Potassium, calcium and magnesium compete with each other in the uptake by the plants (Evangelou et al 1994). Nitrogen fertilization had inconsistent effect on potassium and calcium concentrations in the plant, but generally increases magnesium concentration in the plant (Barker and Pilbeam, 2015). When supplies of cations are plentiful and nitrogen is taken up mainly as nitrate, the plant mineral concentrations may increase owing to the synergistic effect between nitrate and other cation uptake (Whitehead, 2000). The decrement in calcium percentage may be attributed to the increment in potassium owing to the antagonistic effect between each other concentration in the leaves of the plants.

Concerning the phosphorus fertilization rates, the 86 and $98 \mathrm{~kg} \mathrm{P}_{2} \mathrm{O}_{5} /$ feddan application gave the highest percentages of nitrogen, phosphorus, potassium, calcium and magnesium in both seasons, while the 38 and $62 \mathrm{~kg} \mathrm{P}_{2} \mathrm{O}_{5} /$ feddan application gave the lowest percentages of nitrogen, phosphorus, potassium, calcium and magnesium in both seasons. These results are consistent with $\mathbf{L i}$ (2009) on Festival strawberry cultivar that the plant phosphorus accumulation could increase with linear and quadratical effects of plant nitrogen accumulation. The deficiency of phosphorus leads to a lack of energy supply, which can result in limitation in the active uptake of nutrients such as potassium (Barker and Pilbeam, 2015). If phosphorus was applied in conjunction with ammonium, a beneficial interaction occurs. This interaction enhances the plant uptake of both minerals owing to the increase in phosphorus solubility, shoot and root growth (Sumner and Farina, 1986, Bundy et al 2005). Also, positive interactions between $\mathrm{P}$ and $\mathrm{Mg}$ are expected since $\mathrm{Mg}$ is an activator of kinase enzymes and activates most reactions involving phosphate transfer (Hawkesford et al 2012). In addition, phosphorus has antagonistic interaction with calcium in soil due to chemical bonding with phosphorus, for which precipitates are not very soluble, especially in alkaline soils. These precipitates can also occur within roots and other plant tissues, but the acidic biological environment allows for solubilization more readily than with soil (Barker and Pilbeam, 2015).

In addition, the interactive effect of nitrogen and phosphorus fertilization rates on percentages of phosphorus, potassium, calcium and magnesium in leaves were non-significant in both seasons, however it was significant on nitrogen percentage. The treatments which gave the highest nitrogen percentage in leaves were $100 \mathrm{~kg} \mathrm{~N} / \mathrm{feddan}$ with $86 \mathrm{~kg} \mathrm{P}_{2} \mathrm{O}_{5} /$ feddan, $117 \mathrm{~kg} \mathrm{~N} /$ feddan with 74 and

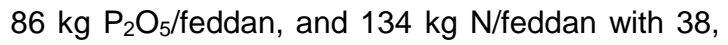
62 and $86 \mathrm{~kg} \mathrm{P}_{2} \mathrm{O}_{5} /$ feddan. 
Table 7. Effect of nitrogen and phosphorus fertilization rates on macronutrient concentrations of strawberry cv. Festival leaves in 2015 and 2016 seasons

\begin{tabular}{|c|c|c|c|c|c|c|c|c|c|c|c|}
\hline & \multirow[b]{2}{*}{ Fertilization rates } & \multicolumn{2}{|c|}{$\mathbf{N}(\%)$} & \multicolumn{2}{|c|}{$\mathbf{P}(\%)$} & \multicolumn{2}{|c|}{$\mathrm{K}(\%)$} & \multicolumn{2}{|c|}{$\mathrm{Ca}(\%)$} & \multicolumn{2}{|c|}{$\mathrm{Mg}(\%)$} \\
\hline & & $\begin{array}{c}1^{\text {st }} \\
\text { season }\end{array}$ & $\begin{array}{c}2^{\text {nd }} \\
\text { season }\end{array}$ & $\begin{array}{c}1^{\text {st }} \\
\text { season }\end{array}$ & $\begin{array}{c}2^{\text {nd }} \\
\text { season }\end{array}$ & $\begin{array}{c}1^{\text {st }} \\
\text { season }\end{array}$ & $\begin{array}{c}2^{\text {nd }} \\
\text { season }\end{array}$ & $\begin{array}{c}1^{\text {st }} \\
\text { season }\end{array}$ & \begin{tabular}{|c|}
$2^{\text {nd }}$ \\
season
\end{tabular} & \begin{tabular}{|c|}
$1^{\text {st }}$ \\
season
\end{tabular} & $\begin{array}{c}2^{\text {nd }} \\
\text { season }\end{array}$ \\
\hline \multicolumn{12}{|c|}{ Nitrogen fertilization } \\
\hline \multicolumn{2}{|r|}{$83 \mathrm{~kg} \mathrm{~N} /$ feddan } & $1.78 \mathrm{c}$ & $1.89 \mathrm{c}$ & $0.305 c$ & $0.315 b$ & $1.63 \mathrm{c}$ & $1.48 \mathrm{~b}$ & $2.16 \mathrm{a}$ & $2.23 \mathrm{a}$ & $0.442 \mathrm{c}$ & $0.525 \mathrm{c}$ \\
\hline \multicolumn{2}{|r|}{$100 \mathrm{~kg} \mathrm{~N} /$ feddan } & $1.95 b$ & $1.99 b$ & $0.331 b$ & $0.315 b$ & $1.66 \mathrm{~b}$ & $1.50 \mathrm{~b}$ & $2.14 \mathrm{a}$ & $2.16 \mathrm{a}$ & $0.519 \mathrm{~b}$ & $0.535 \mathrm{bc}$ \\
\hline \multicolumn{2}{|r|}{$117 \mathrm{~kg} \mathrm{~N} /$ feddan } & $2.00 \mathrm{a}$ & $2.02 \mathrm{ab}$ & $0.351 \mathrm{ab}$ & $0.333 a b$ & $1.71 \mathrm{a}$ & $1.60 \mathrm{a}$ & $2.08 \mathrm{a}$ & $2.12 \mathrm{a}$ & $0.554 \mathrm{a}$ & $0.552 \mathrm{ab}$ \\
\hline \multicolumn{2}{|r|}{$134 \mathrm{~kg} \mathrm{~N} /$ feddan } & $2.05 a$ & $2.08 \mathrm{a}$ & $0.368 \mathrm{a}$ & $0.350 \mathrm{a}$ & $1.74 \mathrm{a}$ & $1.62 \mathrm{a}$ & $2.03 \mathrm{a}$ & $2.10 \mathrm{a}$ & $0.579 \mathrm{a}$ & $0.558 \mathrm{a}$ \\
\hline \multicolumn{12}{|c|}{ Phosphorus fertilization } \\
\hline \multicolumn{2}{|c|}{$38 \mathrm{~kg}$} & $1.85 \mathrm{c}$ & $1.94 \mathrm{~b}$ & $0.311 \mathrm{~d}$ & $0.302 \mathrm{~b}$ & $1.58 \mathrm{c}$ & $1.40 \mathrm{~d}$ & $1.97 \mathrm{c}$ & $2.03 \mathrm{c}$ & $0.497 \mathrm{c}$ & $0.523 \mathrm{c}$ \\
\hline \multicolumn{2}{|r|}{$62 \mathrm{~kg} \mathrm{P}_{2} \mathrm{O}_{5} /$ feddan } & $1.93 b$ & $1.97 b$ & $0.321 \mathrm{~cd}$ & $0.322 a b$ & $1.66 \mathrm{~b}$ & $1.51 \mathrm{c}$ & $2.01 \mathrm{bc}$ & $2.10 \mathrm{bc}$ & $0.501 \mathrm{bc}$ & $0.526 \mathrm{bc}$ \\
\hline \multicolumn{2}{|r|}{$74 \mathrm{~kg} \mathrm{P}_{2} \mathrm{O}_{5} /$ feddan } & $1.96 \mathrm{ab}$ & $1.99 \mathrm{ab}$ & $0.339 \mathrm{bc}$ & $0.331 \mathrm{ab}$ & $1.70 \mathrm{~b}$ & $1.56 \mathrm{~b}$ & $2.06 \mathrm{bc}$ & $2.12 \mathrm{bc}$ & $0.514 \mathrm{bc}$ & $0.544 \mathrm{bc}$ \\
\hline \multicolumn{2}{|r|}{$86 \mathrm{~kg} \mathrm{P}_{2} \mathrm{O}_{5} /$ feddan } & $1.98 \mathrm{ab}$ & $2.06 \mathrm{a}$ & $0.354 a b$ & $0.342 \mathrm{a}$ & $1.74 \mathrm{a}$ & $1.61 \mathrm{a}$ & $2.17 \mathrm{ab}$ & $2.22 \mathrm{ab}$ & $0.540 \mathrm{ab}$ & $0.549 a b$ \\
\hline \multicolumn{2}{|r|}{$98 \mathrm{~kg} \mathrm{P}_{2} \mathrm{O}_{5} /$ feddan } & $2.00 \mathrm{a}$ & $2.01 \mathrm{ab}$ & $0.369 \mathrm{a}$ & $0.346 \mathrm{a}$ & $1.73 \mathrm{a}$ & $1.64 \mathrm{a}$ & $2.29 \mathrm{a}$ & $2.29 \mathrm{a}$ & $0.566 \mathrm{a}$ & $0.569 \mathrm{a}$ \\
\hline \multicolumn{12}{|c|}{ Nitrogen fertilization $\mathrm{X}$ phosphorus fertilization interactions } \\
\hline \multirow{5}{*}{ 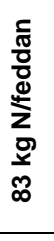 } & $38 \mathrm{~kg} \mathrm{P}_{2} \mathrm{O}_{5} /$ feddan & $1.62 \mathrm{~g}$ & $1.75 \mathrm{~h}$ & $0.273 a$ & $0.285 a$ & $1.54 a$ & $1.36 \mathrm{a}$ & $2.02 a$ & $2.09 a$ & $0.423 a$ & $0.510 a$ \\
\hline & $62 \mathrm{~kg} \mathrm{P} \mathrm{O}_{5} /$ feddan & $1.86 \mathrm{c}-\mathrm{f}$ & $1.99 \mathrm{~d}-\mathrm{g}$ & $0.279 a$ & $0.309 a$ & $1.56 \mathrm{a}$ & $1.42 a$ & $2.09 a$ & $2.20 \mathrm{a}$ & $0.438 \mathrm{a}$ & $0.514 a$ \\
\hline & $74 \mathrm{~kg} \mathrm{P}_{2} \mathrm{O}_{5} /$ feddan & $1.74 \mathrm{fg}$ & $1.85 \mathrm{gh}$ & $0.299 a$ & $0.313 a$ & $1.62 a$ & $1.48 \mathrm{a}$ & $2.13 a$ & $2.24 a$ & $0.444 a$ & $0.529 a$ \\
\hline & $86 \mathrm{~kg} \mathrm{P}_{2} \mathrm{O}_{5} /$ feddan & $1.82 \mathrm{ef}$ & $1.75 \mathrm{~h}$ & $0.328 a$ & $0.333 a$ & $1.70 \mathrm{a}$ & $1.54 a$ & $2.21 \mathrm{a}$ & $2.29 \mathrm{a}$ & $0.449 a$ & $0.534 a$ \\
\hline & $98 \mathrm{~kg} \mathrm{P}_{2} \mathrm{O}_{5} /$ feddan & $1.86 \mathrm{c}-\mathrm{f}$ & 2.09a-e & $0.345 a$ & $0.337 a$ & $1.72 \mathrm{a}$ & $1.60 \mathrm{a}$ & $2.33 a$ & $2.31 \mathrm{a}$ & $0.455 \mathrm{a}$ & $0.538 \mathrm{a}$ \\
\hline \multirow{5}{*}{ 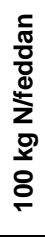 } & $38 \mathrm{~kg} \mathrm{P}_{2} \mathrm{O}_{5} /$ feddan & $1.84 \mathrm{~d}-\mathrm{f}$ & $1.87 \mathrm{gh}$ & $0.325 a$ & $0.296 a$ & $1.58 \mathrm{a}$ & $1.34 a$ & $2.01 a$ & $2.04 a$ & $0.494 a$ & $0.504 a$ \\
\hline & $62 \mathrm{~kg} \mathrm{P}_{2} \mathrm{O}_{5} /$ feddan & $1.89 \mathrm{~b}-\mathrm{e}$ & $1.98 \mathrm{~d}-\mathrm{g}$ & $0.321 a$ & $0.311 \mathrm{a}$ & $1.64 a$ & $1.46 a$ & $2.02 a$ & $2.04 \mathrm{a}$ & $0.478 \mathrm{a}$ & $0.518 a$ \\
\hline & $74 \mathrm{~kg} \mathrm{P} \mathrm{O}_{5} /$ feddan & $2.06 a$ & $2.02 \mathrm{~b}-\mathrm{g}$ & $0.331 a$ & $0.314 a$ & $1.68 \mathrm{a}$ & $1.50 \mathrm{a}$ & $2.13 a$ & $2.09 \mathrm{a}$ & $0.483 a$ & $0.525 a$ \\
\hline & $86 \mathrm{~kg} \mathrm{P}_{2} \mathrm{O}_{5} /$ feddan & $1.94 a-e$ & 2.11a-e & $0.338 a$ & $0.322 a$ & $1.72 \mathrm{a}$ & $1.58 \mathrm{a}$ & $2.24 a$ & $2.22 \mathrm{a}$ & $0.539 a$ & $0.562 a$ \\
\hline & $98 \mathrm{~kg} \mathrm{P}_{2} \mathrm{O}_{5} /$ feddan & $2.01 \mathrm{ab}$ & $1.95 \mathrm{e}-\mathrm{g}$ & $0.339 a$ & $0.333 a$ & $1.70 \mathrm{a}$ & $1.60 \mathrm{a}$ & $2.27 \mathrm{a}$ & $2.40 \mathrm{a}$ & $0.600 \mathrm{a}$ & $0.564 a$ \\
\hline \multirow{5}{*}{ 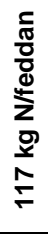 } & $38 \mathrm{~kg} \mathrm{P}_{2} \mathrm{O}_{5} /$ feddan & $1.91 \mathrm{~b}-\mathrm{e}$ & $1.96 \mathrm{e}-\mathrm{g}$ & $0.313 a$ & $0.316 \mathrm{a}$ & $1.60 \mathrm{a}$ & $1.42 \mathrm{a}$ & $1.91 \mathrm{a}$ & $1.93 a$ & $0.524 a$ & $0.534 a$ \\
\hline & $62 \mathrm{~kg} \mathrm{P}_{2} \mathrm{O}_{5} /$ feddan & $1.97 a-d$ & $1.84 \mathrm{gh}$ & $0.326 a$ & $0.323 a$ & $1.72 a$ & $1.58 \mathrm{a}$ & $1.93 a$ & $2.07 \mathrm{a}$ & $0.526 \mathrm{a}$ & $0.538 a$ \\
\hline & $74 \mathrm{~kg} \mathrm{P}_{2} \mathrm{O}_{5} /$ feddan & $1.99 a-c$ & $2.17 a-c$ & $0.358 a$ & $0.336 a$ & $1.74 a$ & $1.66 \mathrm{a}$ & $1.98 a$ & $2.07 \mathrm{a}$ & $0.553 a$ & $0.546 a$ \\
\hline & $86 \mathrm{~kg} \mathrm{P}_{2} \mathrm{O}_{5} /$ feddan & $2.08 \mathrm{a}$ & $2.15 a-d$ & $0.365 a$ & $0.349 a$ & $1.74 a$ & $1.66 a$ & $2.16 a$ & $2.24 a$ & $0.581 \mathrm{a}$ & $0.536 a$ \\
\hline & $98 \mathrm{~kg} \mathrm{P}_{2} \mathrm{O}_{5} /$ feddan & $2.06 \mathrm{a}$ & $1.97 \mathrm{~d}-\mathrm{g}$ & $0.394 a$ & $0.344 a$ & $1.74 \mathrm{a}$ & $1.66 \mathrm{a}$ & $2.40 \mathrm{a}$ & $2.29 \mathrm{a}$ & $0.587 \mathrm{a}$ & $0.603 a$ \\
\hline \multirow{5}{*}{ 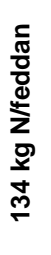 } & $38 \mathrm{~kg} \mathrm{P}_{2} \mathrm{O}_{5} /$ feddan & $2.05 a$ & 2.19ab & $0.331 a$ & $0.311 \mathrm{a}$ & $1.60 \mathrm{a}$ & $1.48 \mathrm{a}$ & $1.93 a$ & $2.07 a$ & $0.546 \mathrm{a}$ & $0.544 a$ \\
\hline & $62 \mathrm{~kg} \mathrm{P}_{2} \mathrm{O}_{5} /$ feddan & $2.00 \mathrm{a}-\mathrm{c}$ & 2.06a-f & $0.356 a$ & $0.345 a$ & $1.74 a$ & $1.60 \mathrm{a}$ & $1.98 \mathrm{a}$ & $2.08 \mathrm{a}$ & $0.562 a$ & $0.534 a$ \\
\hline & $74 \mathrm{~kg} \mathrm{P}_{2} \mathrm{O}_{5} /$ feddan & $2.07 a$ & $1.91 \mathrm{f}-\mathrm{h}$ & $0.368 \mathrm{a}$ & $0.360 a$ & $1.76 \mathrm{a}$ & $1.62 \mathrm{a}$ & $2.00 \mathrm{a}$ & $2.10 \mathrm{a}$ & $0.573 a$ & $0.577 a$ \\
\hline & $86 \mathrm{~kg} \mathrm{P}_{2} \mathrm{O}_{5} /$ feddan & $2.07 a$ & $2.22 \mathrm{a}$ & $0.386 a$ & $0.364 a$ & $1.80 \mathrm{a}$ & $1.68 \mathrm{a}$ & $2.09 a$ & $2.11 \mathrm{a}$ & $0.593 a$ & $0.565 a$ \\
\hline & $98 \mathrm{~kg} \mathrm{P}_{2} \mathrm{O}_{5} /$ feddan & $2.06 a$ & $2.02 \mathrm{c}-\mathrm{g}$ & $0.397 a$ & $0.371 \mathrm{a}$ & $1.78 \mathrm{a}$ & $1.70 \mathrm{a}$ & $2.16 a$ & $2.14 a$ & $0.623 a$ & $0.570 \mathrm{a}$ \\
\hline
\end{tabular}

Means into every group within a column for the same factor followed by the same letter are not significantly different $(P \leq 0.05)$ according to Duncan's multiple range test. 
Increasing the fertilization rates of nitrogen and phosphorus increased the mineral contents in leaves of strawberry plants. This may be attributed to the transplant vigor and the increase of the vegetative growth, i.e. root length, plant length, number of leaves/plant, average leaf area, fresh and dry weights of roots and vegetative growth, as mentioned in Tables (2 and 3), also the increase in the crown diameter and crown total carbohydrates and SPAD reading as mentioned in Tables (4 and 6).

\section{CONCLUSION}

Nitrogen and phosphorus are the main nutrients that affect the number and quality of strawberry transplants in the nurseries. It is the first scientific research addressing the optimal requirements of both elements for strawberry nurseries in Egypt. The current study demonstrated that increasing the rates of nitrogen and phosphorus fertilizations increased the number of main runners/mother plant, number of marketable transplants $/ \mathrm{m}^{2}$, and all quality parameters of the produced transplants, recommending to the nurserymen of Festival strawberry cultivar to apply 117 or $134 \mathrm{~kg} \mathrm{~N}$ with $98 \mathrm{~kg} \mathrm{\textrm {P } _ { 2 } \mathrm { O } _ { 5 }}$ per feddan in pure sandy soil which gave the highest number of transplants with high quality and subsequent high quality crop in the field.

\section{Conflict of interest}

The authors declare that they have no conflict of interest.

\section{REFERENCES}

Acuña-Maldonado, L.E. and Pritts, M.P. 2008. Carbon and nitrogen reserves in perennial strawberry affect plant growth and yield. J. Amer. Soc. Hort. Sci., 133(6), 735-742.

Aloni, B., Pashkar, T., Karni, L. and Daie, J. 1991. Nitrogen supply influences carbohydrate partitioning of pepper seedlings and transplant development. J. Amer. Soc. Hort. Sci., 116(6), 995-999.

Alpert, P. 1991. Nitrogen sharing among ramets increases clonal growth in Fragaria chiloensis. Ecology, 72(1), 69-80. doi: 10.2307/1938903.

AOAC, 2005. Association of Official Analytical Chemists-International, Official Methods of Analysis, $18^{\text {th }}$, Horwitz W. and Latimer G.W. (eds.), 107 p., AOAC-Int. Suite 500, 481 North Frederick Avenue, Gaithersburg, Maryland, USA.
Barker, A.V. and Pilbeam, D.J. 2015. Handbook of plant nutrition, $2^{\text {nd }}$ Ed. 17 p., CRC Press, Taylor and Francis, Boca Raton, Florida, USA.

Barrs. H.D. and Weatherley, P.E. 1962. A reexamination of the relative turgidity technique for estimating water deficits in leaves. Aust. J. Biol. Sci., 15(3), 413-428. doi: 10.1071/BI9620413.

Basoccu, L. and Nicola, S. 1995. Supplementary light and pretransplant nitrogen effects on tomato seedling growth and yield. Acta Hortic., 396, 313-320. doi: 10.17660/ActaHortic.1995.396.37.

Black, C.A., Evans, D.D., White, J.L., Ensminger, L.E., Clark, F.E. and Dinauer, R.C. 1965. Methods of soil analysis II: chemical and microbiological properties. 103 p., Amer. Soc. Agron. Inc., Madison, Wisconsin, USA.

Blatt, C.R. and Crouse, D.N.A. 1970. Effects of gibberellic acid and nitrogen on the strawberry cv. 'Redcoat'. HortScience, 5, 437-438.

Bloom, A.J. 2015. The increasing importance of distinguishing among plant nitrogen sources. Current Opinion in Plant Biology, 25, 10-16. doi: 10.1016/j.pbi.2015.03.002.

Bundy, L.G., Tunney, H. and Halvarson, A.D. 2005. Agronomic aspects of phosphorus management. In: Sims J.T. and Sharpley A.N. (eds.), Phosphorus: Agriculture and the Environment, pp. 685-727, American Society of Agronomy, Crop Science Society of America, Soil Science Society of America. Madison, WI, USA.

Carrillo-Mendoza, O., Rodríguez-Alcázar, J., Cano-Medrano, R. and López-Jiménez, A. 2005. Foliar application of urea and sucrose, their effects on nursery plant conditioning and production of strawberry (Fragaria $\times$ ananassa Duch.) 2 'CP 99-3A'. Agrociencia, 39(2), 195204.

Chapman, H.D. and Pratt, P.F. 1961. Methods of Analysis for Soils, Plants, and Waters. 33 p., Division of Agriculture Sciences, University of California, Berkeley, CA, USA.

Chapman, S.C. and Barreto, H.J. 1997. Using a chlorophyll meter to estimate specific leaf nitrogen of tropical maize during vegetative growth. Agronomy J., 89, 557-562. doi:10.2134/agronj1997.00021962008900040 004x. 
Cheng, K.L. and Bray, R.H. 1951. Determination of calcium and magnesium in soil and plants materials. Soil Sci., 72(6), 449-458.

Choi, J.M. and Lee, C.W. 2012. Influence of elevated phosphorus levels in nutrient solution on micronutrient uptake and deficiency symptom development in strawberry cultured with fertigation system. J. of Plant Nutrition, 35(9), 13491358. doi: 10.1080/01904167.2012.684127

Choi, J.M., Latigui, A. and Lee, C.W. 2013. Visual symptom and tissue nutrient contents in dry matter and petiole sap for diagnostic criteria of phosphorus nutrition for 'Seolhyang' strawberry cultivation. Hort., Environ. Biotechnol., 54(1), 52-57. doi: 10.1007/s13580-013-0130-y.

De Long, J.R., Sundqvist M.K., Gundale M.J., Giesler, R. and Wardle, D.A. 2016. Effects of elevation and nitrogen and phosphorus fertilization on plant defence compounds in subarctic tundra heath vegetation. Funct. Ecol., 30, 314325. doi: 10.1111/1365-2435.12493.

Deng, X. and Woodward, F.I. 1998. The growth and yield responses of Fragaria ananassa to elevated CO2 and N Supply. Annals of Botany, 81, 67-71.

Druege, U., Zerche, S. and Kadner, R. 2004. Nitrogen- and storage-affected carbohydrate partitioning in high-light-adapted Pelargonium cuttings in relation to survival and adventitious root formation under low light. Annals of Botany, 94, 831-842. doi: 10.1093/aob/mch210.

DuBois, M., Gilles, K.A., Hamilton, J.K., Rebers, P.A. and Smith, F. 1956. Colorimetric method for determination of sugars and related substances. Analytical Chemistry, 28(3), 350356. doi: 10.1021/ac60111a017

Dufault, R.J. and Schultheis, J.R. 1994. Bell pepper seedling growth and yield following pretransplant nutritional conditioning. HortScience, 29(9), 999-1001.

Epstein, E. and Bloom A.J. 2004. Mineral Nutrition of Plants: Principles and perspectives. $\left(2^{\text {nd }}\right.$ Ed.), 28 p., John wiley and Sons, Inc., New York, USA.

Evangelou, V.P., Wang, J. and Phillips, R.E. 1994. New developments and perspectives on soil potassium quantity/intensity relationships. Advances in Agronomy, 52, 173-227.

FAOSTAT 2014. Food and Agriculture organizations of the United Nations. Statistics Division. http://www.fao.org/faostat/en/\#data/QC (accessed 15, October 2017).
García-Méndez, E., García-Sinovas, D., Andrade, M.A., González, B., Sánchez-Sevilla, J.F., López-Aranda, J.M., Medina, J.J. and Miranda, L. 2009. Influence of nitrogen fertilization and digging date on strawberry plant maturity. Acta Hortic., 842, 687-690. doi: 10.17660/ActaHortic.2009.842.148

Grevsen, K., Frette, X.C., and Christensen, L.P. 2008. Concentration and composition of flavonol glycosides and phenolic acids in aerial parts of stinging nettle (Urtica dioila L.) are affected by nitrogen fertilization and by harvest time. European J. of Horticultural Science, 73(1), 20-27.

Hawkesford, M., Horst, W., Kichey, T., Lambers, H., Schjoerring, J., Møller, I.S. and White P. 2012. Functions of macronutrients. In: Marschner, H. (ed.), Marschner's Mineral Nutrition of Higher Plants. ( $3^{\text {rd }}$ Ed.), 135 p., Academic Press, Inc., London, United Kingdom.

Heil, M. and Baldwin I.T. 2002. Fitness costs of induced resistance: Emerging experimental support for a slippery concept. Trends Plant Sci., 7, 61-67. doi:10.1016/S13601385(01)02186-0

Hermans, C., Hammond, J.P., White, P.J. and Verbruggen, N. 2006. How do plants respond to nutrient shortage by biomass allocation?. Trends in Plant Science, 11(12), 610-617. doi: 10.1016/j.tplants.2006.10.007

Herms, D.A. and Mattson, W.J. 1992. The dilemma of plants: to grow or defend. The Quarterly Review of Biology, 67(3), 283-335. doi: 10.1086/417659

Hill, J.O., Simpson, R.J., Moore, A.D. and Chapman, D.F. 2006. Morphology and response of roots of pasture species to phosphorus and nitrogen nutrition. Plant and Soil, 286, 7-19. doi: 10.1007/s11104-006-0014-3.

Jackson, M.L. 1973. Soil chemical analysis. 10 p., Prentice-Hall of India Pvt. Ltd., New Delhi, India.

Jang, S.W., Hamayun, M., Sohn, E.Y., Shin, D.H., Kim K.U., Lee B.H. and Lee, I.J. 2008. Effect of elevated nitrogen levels on endogenous gibberellin and jasmonic acid contents in three rice (Oryza sativa L.) cultivars. J. Plant Nutr. Soil Sci., 171, 181-186. doi: 10.1002/jpln.200625025.

Janisch, D.I., Andriolo, J.L., Toso, V., Ferreira dos Santos, K.G. and Maronez de Souza, J. 2012. Nitrogen for growth of stock plants and production of strawberry runner tips. Bra- 
gantia, Campinas, 71(3), 394-399. doi: 10.1590/S0006-87052012005000027

Jones, C.G. and Hartley, S.E. 1999. A protein competition model of phenolic allocation. Oikos, 86(1), 27-44. doi: 10.2307/3546567

Karchi, Z., Dagan, A. and Cantliffe, D.J. 1992. Growth of containerized lettuce transplants supplemented with varying concentrations of nitrogen and phosphorus. Acta Hortic., 319, 365-370.

doi:

10.17660/ActaHortic.1992.319.56

Khan, W., Prithviraj, B. and Smith, D.L. 2003. Photosynthetic responses of corn and soybean to foliar application of salicylates. J. Plant Physiol., 160(5), 485-492. doi: 10.1078/01761617-00865

Kirschbaum, D.S., Larson, K.D., Weinbaum, S.A. and DeJong, T.M. 2010. Late-season nitrogen applications in high-latitude strawberry nurseries improve transplant production pattern in warm regions. Afr. J. Biotechnol., 9(7), 1001-1007. doi: 10.5897/AJB09.1059

Kirschbaum, D.S., Larson, K.D., Weinbaum, S.A. and DeJong, T.M. 2015. Differential response of early and intermediate flowering strawberry cultivars to nursery late-season nitrogen applications and digging date. Afr. J. Plant Sci., 9(6), 250-263. doi: 10.5897/AJPS2015.1280

Koller, H.R.C. 1972. Leaf area-leaf weight relationships in the soybean canopy. Crop Sci., 12(2), 180-183. doi: 10.2135/cropsci 1972.0011183X001200020007x

Koricheva, J. 2002. Meta-analysis of sources of variation in fitness costs of plant antiherbivore defenses. Ecology, 83(1), 176-190.

Leghari, S.J., Wahocho, N.A., Laghari, G.M., Laghari, A.H., Bhabhan, G.M., Talpur, K.H., Bhutto, T.A., Wahocho, S.A. and Lashari, A.A. 2016. Role of Nitrogen for Plant Growth and Development: A review. Advances in Environmental Biology, 10(9), 209-218.

Li, H. 2009. Strawberry Plant Propagation, Potential Phosphorus Pool and Factors Influencing Phosphorus Use Efficiency in Nursery Systems. Final Report, pp. 1-30, North American Strawberry Growers Association Research Program.

Li, H., Li, T., Gordon, R.J. and Asiedu, S. 2009. Strawberry nursery plant propagation in relation to soil phosphorus and water variation. In 'Proc. $16^{\text {th }}$ International Plant Nutrition Colloquium.
Functions, Interactions and Diagnosis of Nutrient Status', pp. 17-19, Dept., of Plant Sciences, UC Davis, California, USA.

Li, H., Huang, R. Li, T. and Hu, K. 2010. Ability of nitrogen and phosphorus assimilation of seven strawberry cultivars in a northern Atlantic coastal soil. In: $19^{\text {th }}$ World Congress of Soil Sci., Soil Solutions for a Changing World, pp. 1-6 August 2010, Brisbane, Australia.

Li, H., Li, T., Fu, G. and Hu, K. 2014. How strawberry plants cope with limited phosphorus supply: Nursery-crop formation and phosphorus and nitrogen uptake dynamics. J. Plant Nutr. Soil Sci., 177, 260-270. doi: 10.1002/jpln.201200654

Liptay, A., Nicholls, S. and Sikkema, P. 1992. Optimal mineral nutrition of tomato transplants in the greenhouse for maximum performance in the field. Acta Hortic., 319, 489-492. doi: 10.17660/ActaHortic.1992.319.76

López-Arnaldos, T., Muñoz R., Ferrer, M.A. and Calderón, A.A. 2001. Changes in phenol content during strawberry (Fragaria $x$ ananasa, cv. Chandler) callus culture. Physiol. Plant., 113(3), 315-322.

Lu, Z.X., Villareal, S., Yu, X.P., Heong, K.L. and Hu, C. 2004. Effect of nitrogen on water content, sap flow and tolerance of rice plants to brown plant hopper. Rice Sci., 11(3), 129-134.

Maas, J.L., Wang, S.Y. and Galletta, G.J. 1991. Evaluation of strawberry cultivars for allegic acid content. HortScience, 26(1), 66-68.

Macías-Rodríguez, L., Quero, E. and López, M.G. 2002. Carbohydrate differences in strawberry crowns and fruit (Fragaria $x$ ananassa) during plant development. J. Agric. Food Chem., 50(11), 3317-3321.

Mandal, K., Saravanan, R. and Maiti, S. 2008. Effect of different levels of $\mathrm{N}, \mathrm{P}$ and $\mathrm{K}$ on downy mildew (Peronospora planthginis) and seed yield of Isabgol (Plantago ovata). Crop Protection, 27(6), 988-995. doi: 10.1016/j.cropro.2007.12.002

Marschner, H. 2012. Marschner's Mineral Nutrition of Higher Plants. ( $3^{\text {rd }}$ Ed.), 3 p. Academic Press, Inc., London, United Kingdom.

Massad, T.J., Dyer, L.A. and Vega, C.G. 2012. Costs of defense and a test of the carbonnutrient balance and growth-differentiation balance hypotheses for two co-occurring classes of plant defense. PLOS ONE, 7(10), 1-8. e47554. doi:10.1371/journal.pone.0047554 
Melton, R.R. and Dufault, R.J. 1991. Nitrogen, phosphorus, and potassium fertility regimes affect tomato transplant growth. HortScience, 26(2), 141-142.

Naghashzadeh, M. 2014. Response of relative water content and cell membrane stability to mycorrhizal biofertilizer in maize. Electronic $\mathbf{J}$. of Biology, 10(3), 68-72.

Nam, M.H., Jeong, S.K., Lee, Y.S., Choi, J.M. and Kim, H.G. 2006. Effects of nitrogen, phosphorus, potassium and calcium nutrition on strawberry anthracnose. Plant Pathology, 55, 246-249. doi: 10.1111/j.13653059.2005.01322.x

Namvar, A. and Khandan, T. 2015. Inoculation of rapeseed under different rates of inorganic nitrogen and sulfur fertilizer: impact on water relations, cell membrane stability, chlorophyll content and yield. Archives of Agronomy and Soil Sci., 61(8), 1137-1149. doi: 10.1080/03650340.2014.982550

Nguyen, P.M. and Niemeyer, E.D. 2008. Effects of nitrogen fertilization on the phenolic composition and antioxidant properties of basil (Ocimum basilicum L.). J. Agric. Food Chem., 56(18), 8685-8691. doi: 10.1021/ jf801485u.

Page, A.L., Miller, R.H. and Keeney, D.R. 1982. Methods of soil analysis-chemical and microbiology properties, Part 2. 228 p., SSSA Inc., Mad., WI., USA.

Ragab, M.I. 1996. Effect of GA3 on number and some transplant characters of strawberry nurseries. Fourth Arab Conf., Minia Soc. Hort. Sci., 78, 261-269.

Rodgers, C.O., Izsak, E., Kafkafi, U. and Izhar, S. 1985. Nitrogen rates in strawberry (Fragaria ananasa) nursery on growth and yield in the field. J. of Plant Nutrition, 8(2), 147-162. doi: 10.1080/01904168509363331

Sairam, R.K., Deshmukh, P.S. and Shukla, D.S. 1997. Tolerance of drought and temperature stress in relation to increased antioxidant enzyme activity in wheat. J. Agron. Crop Sci., 178(3), 171-178. doi: 10.1111/j.1439-037X. 1997.tb00486.x

Singh, V., Pallaghy, C.K., and Singh, D. 2006. Phosphorus nutrition and tolerance of cotton to water stress II. Water relations, free and bound water and leaf expansion rate. Field Crops Research, 96, 199-206. doi: 10.1016/ j.fcr.2005.06.011

Sousa, C., Pereira, D.M., Pereira, J.A., Bento, A., Rodrigues, M.A., Dopico-García, S., Valentão, P., Lopes, G., Ferreres, F., Seabra, R.M. and Andrade, P.B. 2008. Multivariate analysis of tronchuda cabbage (Brassica oleracea L. var. costata DC) phenolics: influence of fertilizers. J. Agric. Food Chem., 56(6), 2231-2239. doi: 10.1021/jf0730410

Sumner, M.E. and Farina, M.P.W. 1986. Phosphorus interactions with other nutrients and lime in field cropping systems. Adv. Soil Sci., 5, 201-236.

Thomas, R.L., Sheard, R.W. and Moyer, J.R. 1967. Comparison of conventional and automated procedures for nitrogen, phosphorus and potassium analysis of plant materials using a single digestion. Agronomy J., 59(3), 240243.doi:10.2134/agronj1967.00021962005900 030010x

Tworkoski, T.J., Benassi, T.E. and Takeda, F. 2001. The effect of nitrogen on stolon and ramet growth in four genotypes of Fragaria chiloensis L. Scientia Horticulturae, 88(2), 97106. doi: 10.1016/S0304-4238(00)00198-9

Uchida, R. 2000. Essential Nutrients for Plant Growth: Nutrient Functions and Deficiency Symptoms. In: J. A. Silva and R. Uchida (eds.), Plant Nutrient Management in Hawaii's Soils, Approaches for Tropical and Subtropical Agric.. pp. 31-55, College of Tropical Agric. and Human Resources, Univ., of Hawaii, Mānoa, Honolulu, Hawaii, USA..

Verpont, F. 2003. Quality of strawberry plants. Effect of nitrogen fertilization in the nursery. Infos-Ctifl, 188, 41-45.

Waller, R.A. and Duncan, D.B. 1969. A Bayes rule for the symmetric multiple comparison problem. J. of the American Statistical Association, 64(328), 1484-1503. doi: $10.2307 / 2286085$

Wang, B., Chu, J., Yu, T., Xu, Q., Sun, X., Yuan, J., Xiong, G., Wang, G., Wang, Y. and Li, J. 2015. Tryptophan-independent auxin biosynthesis contributes to early embryogenesis in Arabidopsis. Proc. Natl. Acad. Sci. USA, 112(15), 4821-4826. doi: 10.1073/pnas. 1503998112

Whitehead, D.C. 2000. Nutrient elements in grassland Soil-plant-animal relationships. 66 p., CABl publishing.

Zhang, J., Wang, Y., Wang, P., Zhang, Q., Yan, C., Yu, F., Yi, J. and Fang, L. 2017. Effect of different levels of nitrogen, phosphorus, and potassium on root activity and chlorophyll content in leaves of Brassica oleracea seedlings grown in vegetable nursery substrate. Hortic., Environ. Biotechnol., 58(1), 5-11. doi: 10.1007/s13580-017-0177-2

Zhao, Y. 2012. Auxin biosynthesis: a simple twostep pathway converts tryptophan to indole-3acetic acid in plants. Molecular Plant, 5(2), 334-338. doi:10.1093/mp/ssr104. 
Arab Univ. J. Agric. Sci., Ain Shams Univ., Cairo Special Issue, 26(2A), 951- 969, 2018 